\title{
Mineralogy and geochemistry of granitoids from Kinnaur region, Himachal Higher Himalaya, India: Implication on the nature of felsic magmatism in the collision tectonics
}

\author{
Brajesh Singh ${ }^{1}$, Santosh Kumar ${ }^{1, *}$, Masao Ban ${ }^{2}$ and Kazuo Nakashima ${ }^{2}$ \\ ${ }^{1}$ Department of Geology, Kumaun University, Nainital 263 002, Uttarakhand, India. \\ ${ }^{2}$ Department of Earth and Environmental Sciences, Yamagata University, 990 8560, Yamagata, Japan. \\ *Corresponding author. e-mail: skyadavan@yahoo.com
}

Felsic magmatism in the southern part of Himachal Higher Himalaya is constituted by Neoproterozoic granite gneiss (GGn), Early Palaeozoic granitoids (EPG) and Tertiary tourmaline-bearing leucogranite (TLg). Magnetic susceptibility values $\left(<3 \times 10^{-3} \mathrm{SI}\right)$, molar $\mathrm{Al}_{2} \mathrm{O}_{3} /\left(\mathrm{CaO}+\mathrm{Na}_{2} \mathrm{O}+\mathrm{K}_{2} \mathrm{O}\right)(\geq 1.1)$, mineral assemblage (bt-ms-pl-kf-qtz \pm tur \pm ap), and the presence of normative corundum relate these granitoids to peraluminous S-type, ilmenite series (reduced type) granites formed in a syncollisional tectonic setting. Plagioclase from GGn $\left(\mathrm{An}_{10}-\mathrm{An}_{31}\right)$ and EPG $\left(\mathrm{An}_{15}-\mathrm{An}_{33}\right)$ represents oligoclase to andesine and TLg $\left(\mathrm{An}_{2}-\mathrm{An}_{15}\right)$ represents albite to oligoclase, whereas compositional ranges of K-feldspar are moreor-less similar ( $\mathrm{Or}_{88}$ to $\mathrm{Or}_{95}$ in GGn, $\mathrm{Or}_{86}$ to $\mathrm{Or}_{97}$ in EPG and $\mathrm{Or}_{87}$ to $\mathrm{Or}_{94}$ in TLg). Biotites in GGn $\left(\mathrm{Mg} / \mathrm{Mg}+\mathrm{Fe}^{\mathrm{t}}=0.34-0.45\right), \mathrm{EPG}\left(\mathrm{Mg} / \mathrm{Mg}+\mathrm{Fe}^{\mathrm{t}}=0.27-0.47\right)$, and $\mathrm{TLg}\left(\mathrm{Mg} / \mathrm{Mg}+\mathrm{Fe}^{\mathrm{t}}=0.25-0.30\right)$ are ferribiotites enriched in siderophyllite, which stabilised between FMQ and HM buffers and are characterised by dominant $3 \mathrm{Fe} \rightleftharpoons 2 \mathrm{Al}, 3 \mathrm{Mg} \rightleftharpoons 2 \mathrm{Al}$ substitutions typical of peraluminous (S-type), reducing felsic melts. Muscovite in GGn $\left(\mathrm{Mg} / \mathrm{Mg}+\mathrm{Fe}^{\mathrm{t}}=0.58-0.66\right)$, EPG $\left(\mathrm{Mg} / \mathrm{Mg}+\mathrm{Fe}^{\mathrm{t}}=0.31-0.59\right)$, and $\mathrm{TLg}$ $\left(\mathrm{Mg} / \mathrm{Mg}+\mathrm{Fe}^{\mathrm{t}}=0.29-0.42\right)$ represent celadonite and paragonite solid solutions, and the tourmaline from EPG and TLg belongs to the schorl-elbaite series, which are characteristics of peraluminous, Li-poor, biotite-tourmaline granites. Geochemical features reveal that the GGn and EPG precursor melts were most likely derived from melting of biotite-rich metapelite and metagraywacke sources, whereas TLg melt appears to have formed from biotite-muscovite rich metapelite and metagraywacke sources. Major and trace elements modelling suggest that the GGn, EPG and TLg parental melts have experienced low degrees $(\sim 13, \sim 17$ and $\sim 13 \%$, respectively) of kf-pl-bt fractionation, respectively, subsequent to partial melting. The GGn and EPG melts are the results of a pre-Himalayan, syn-collisional Pan-African felsic magmatic event, whereas the TLg is a magmatic product of Himalayan collision tectonics.

\section{Introduction}

The Himalayan Metamorphic Belt (HMB) in Himachal Himalaya is comprised of Higher Himalayan Crystalline (HHC) rocks in the north, and Lesser Himalayan rocks are exposed in numerous windows that form the lower margin of the HMB.
The HHC acts as the basement for the Phanerozoic Tethyan sedimentary pile (Bhargava et al. 1991) and is mainly composed of Palaeoproterozoic to Early Palaeozoic metasediments (pelitic and psammitic) and felsic magmatic rocks (400-500, 1200-1400 and 1800-2000 Ma), which are the reworked protoliths to the Neogene leucogranites

Keywords. Mineralogy; geochemistry; petrogenesis; granitoids; Himachal Higher Himalaya. 
(14-28 Ma, Rb-Sr whole rock isochron; Srikantia and Bhargava 1998) formed during the Himalayan collision event (Gansser 1964; Le Fort 1975; Srikantia and Bhargava 1998). The HHC in the Kinnaur district of the Himachal Himalaya forms the Vaikrita Group, and is mainly represented by Neoproterozoic (601 $\pm 19 \mathrm{Ma}$; Rb-Sr whole rock isochron; Mehta 1977) granite gneiss (GGn), intercalated metasedimentary rocks and Early Palaeozoic $(474 \pm 26 \mathrm{Ma}$; $\mathrm{Rb}-\mathrm{Sr}$ whole rock isochron; Kwatra et al. 1999; Singh and Kumar 2005) granitoids (EPG), which are later intruded by Tertiary tourmaline-bearing leucogranites (TLg). The Palaeoproterozoic to Mesoproterozoic granitoids are, however, not exposed in the study area.

The EPG in the Indian Himalaya region represent a Pan-African tectonothermal event that occurred around the Cambro-Ordovician boundary, and are extensively exposed in the Crystalline Thrust Sheets of the Lesser Himalaya in the HHC and in the metamorphosed basal portions of the Tethyan sedimentary rocks (Kumar et al. 1996; Islam and Gururajan 2003). The EPG have mineralogical and geochemical features that are identical to syn-collisional, peraluminous granitoids with an elevated initial $\mathrm{Sr}$-isotopic ratio $\left(\mathrm{Sr}_{\mathrm{i}}=0.706-0.770\right.$; Le Fort et al. 1986a, b).

The geology and structure of the Kinnaur basin have been described by many workers (e.g., Sharma 1976; Bhargava 1982; Bassi and Chopra 1983; Kakar 1988; Mishra 1993; Srikantia and Bhargava 1998). Geochemistry and whole-rock Rb-Sr geochronology of the EPG from the Satluj-Baspa valleys of Himachal Himalaya have been previously discussed (Kwatra et al. 1999; Islam and Gururajan 2003). Based on Rb-Sr whole rock isotopic data, Kwatra et al. (1999) have suggested two discrete felsic magmatic pulses as coarse grained Akpa granitoids $(477 \pm 29 \mathrm{Ma}$; Sri $=0.721)$ and medium grained Rakcham granitoids (453 \pm 9 Ma; Sri $=0.737)$, both belonging to the Early Palaeozoic granitoids. However, contact relationships between these granitoid bodies cannot be established, and it is likely that both the granitoids are products of a single magmatic event that occurred at $474 \pm 26 \mathrm{Ma}$ (age calculated combining $\mathrm{Rb}-\mathrm{Sr}$ data of Akpa and Rakcham granitoids by Singh and Kumar 2005). Based on published (Kwatra et al. 1999) and new database, a petrogenetic reappraisal of EPG from the Kinnaur region has been proposed by Singh and Kumar (2005). In this paper, we provide comprehensive field relations, measurements of magnetic susceptibility, petrography, mineral and whole-rock geochemistry of GGn, EPG and TLg from Kinnaur region, and using this we propose a viable petrogenetic model for the evolution of felsic magmatism in the collisional tectonic environment of the area.

\section{Geology and field relation}

The study area forms a part of the HHC, erstwhile Central Crystalline Zone (Srikantia and Bhargava 1998), of the Vaikrita Group located in the southern part of Higher Himachal Himalaya (figure 1a). The studied lithological units are best exposed along the Satluj and Baspa (Sangla) river valleys and their tributaries in, and around the Reckong Peo, Ribba, Akpa, Karcham, Rakcham and Chitkul areas and beyond (figure 1b). Some parts of these regions consist of metasedimentary rocks, migmatites and a variety of Neoproterozoic gneisses (psammitic gneiss, banded gneiss, granite gneiss, coarse psammitic gneiss; Kakar 1988; Bartarya et al. 1996; Gupta et al. 2001) collectively referred herein as granitic gneiss (GGn). They are spatially associated with Early Palaeozoic granitoids (EPG) and tourmaline-bearing leucogranites (TLg) of the Haimanta Group. The GGn and EPG are thrust over the Mesoproterozoic to Neoproterozoic Karcham Group of rocks (三Chail Group), which are largely composed of mica schist, gneiss and quartzite. These units are further thrust over the Jutogh Group which comprises the Wangtu Gneissic Complex.

The GGn show well-developed, leucocratic to mesocratic bands of variable thickness. K-feldspar dominates in the leucocratic bands, where it is elongated and exhibits a preferred orientation. Melanocratic bands are dominated by biotite. In places, augen (quartz and feldspar) follow the gneissosity but are randomly oriented. The GGn exhibit in situ leucocratic layers that have melanocratic borders suggesting the formation of localised migmatites close to a shear zone (trending $\mathrm{N} 35^{\circ} \mathrm{W}-$ $\mathrm{S} 35^{\circ} \mathrm{E}$ and dipping $60^{\circ}-70^{\circ}$ towards $\mathrm{NE}$ ) near Purbani village, which contains skarns (scheelite), pyrite, chalcopyrite, pyrrhotite and bornite mineralisation. The magnetic susceptibility (MS) values of GGn range from 0.052 to $0.220 \times 10^{-3}$ SI units with an average MS value of $0.156 \times 10^{-3}$ SI units (figure 2).

The EPG are fine- to coarse-grained, mostly porphyritic, two-mica (muscovite and biotite) granite (sensu stricto) that range from foliated to nonfoliated varieties and show well-developed joints and fractures. Near the contact with the country rocks, the EPG are moderately foliated and exhibit a mylonitic structure, and in and around Ribba village, these exhibit leucocratic to melanocratic bands. Euhedral to anhedral felsic megacrysts composed of quartz and feldspar are common in the porphyritic varieties. In and around Rakcham village, the EPG exhibit schlieren-like, magmatic flow structures that are defined by mafic minerals. The EPG have sharp contacts with the Tethyan sequence near Akpa village. Enclaves in 
(a)

(b)

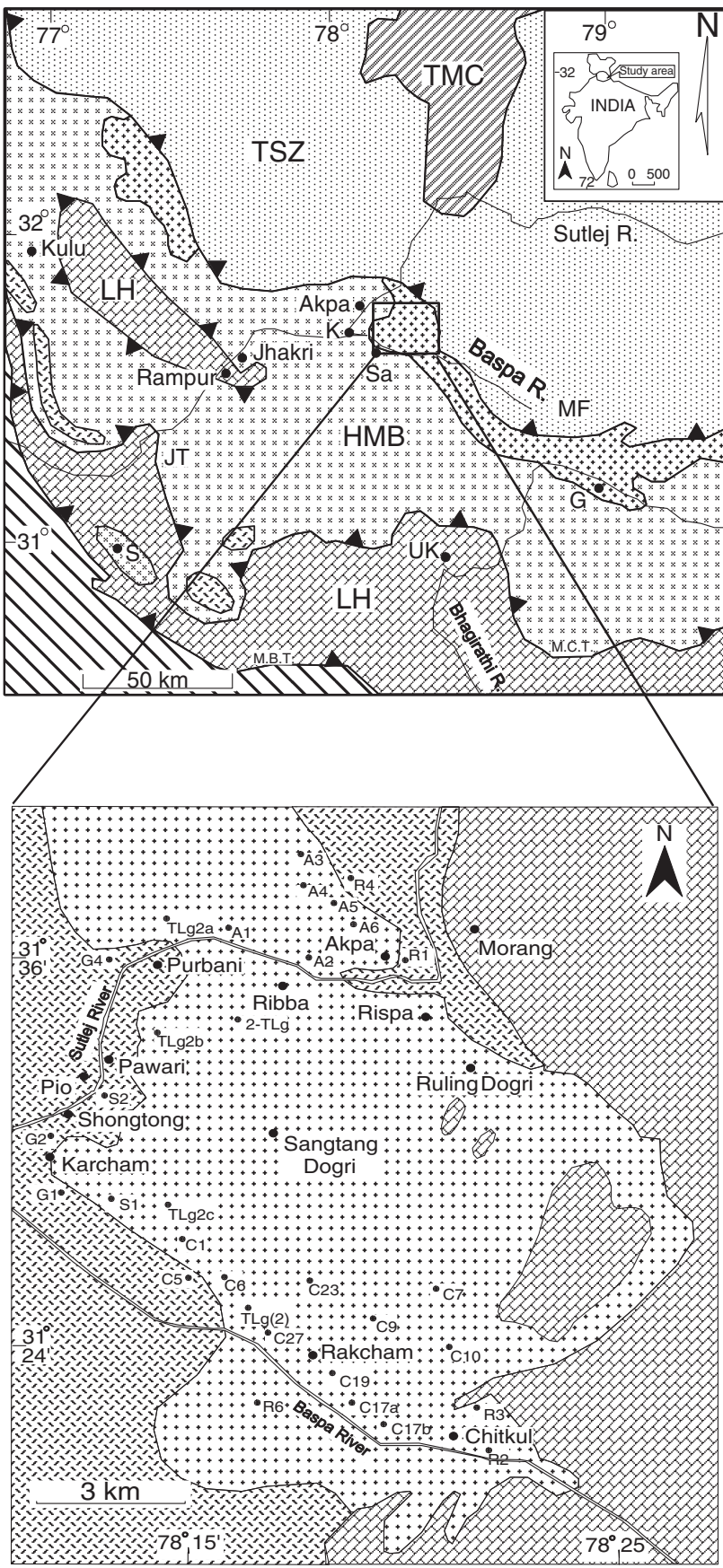

LEGEND

N Sub-Himalayan Cenozoic

Sedimentary Foreland

Lesser Himalaya (LH)

Proterozoic Sedimentary Sequence

Pan-African biotite granite/gneiss \&

mid-Cenozoic Anatectic Leucogranite

Lesser Himalaya ca $500 \mathrm{Ma}$

Granitoid Belt

Himalayan Metamorphic Belt (HMB)

Tethyan Sedimentary Zone (TSZ)

Tso-Morari Crystallines (TMC)

Study area

MBT- Main Boundary Thrust;

MCT- Main Central Thrust;

JT- Jutogh Thrust; MF- Martoli Fault;

S- Shimla; K- Karcham; Sa- Sangla;

UK- Uttarkashi; G- Gangotri

INDEX

ZYZ Tethyan Sedimentary Zone (TSZ)

\# * * Early Palaeozoic Granitoids (EPG)

: * * (Akpa / Rakcham)

Vaikrita Group (garnet or staurolite or

$\therefore$ kyanite or sillimanite schist and/or gneiss, calc-silicates, augen gneiss, migmatite)

3

Analyzed Sample Location and Number

Figure 1. (a) Tectonic framework of Himalayan collision zone of NW Himalaya (after Kwatra et al. 1999) and (b) geological map of study area (after Kwatra et al. 1999) showing the occurrence of Early Palaeozoic granitoids (EPG) and associated units. Tourmaline bearing leucogranite $(\mathrm{TLg})$ is not mappable at present scale shown as intruding the EPG body.

the EPG are relatively rare and represent either surmicaceous material (biotite schists), xenoliths of country rocks (phyllite, biotite schist, gneiss, psammite), or are of felsic magmatic origin. The surmicaceous enclave (SE) is rounded to elliptical $(20 \times 5 \mathrm{~cm})$ in shape and shows sharp contacts with its felsic host. Country rock xenoliths (CRX) are angular to rounded in shape, large in size (up to $1 \mathrm{~m}$ across) and closely resemble the older GGn unit, but show reacted margins. At places, quartz veins and TLg cross the xenoliths and the host
EPG. The felsic magmatic enclaves (FME) are fine-grained, rounded to lenticular in shape (up to $70 \mathrm{~cm}$ across) and exhibit sharp to partially diffuse contacts, as can be observed in and around the Akpa village. The EPG have MS values ranging from 0.016 to $0.187 \times 10^{-3}$ SI (figure 2), the average $(\mathrm{N}=104)$ for the MS being $\chi=0.141 \times 10^{-3} \mathrm{SI}$ (Singh and Kumar 2005).

Bands of tourmaline-bearing leucogranite (TLg) range in width from a few meters to about $250 \mathrm{~m}$ and commonly intrude the EPG and GGn, mostly 


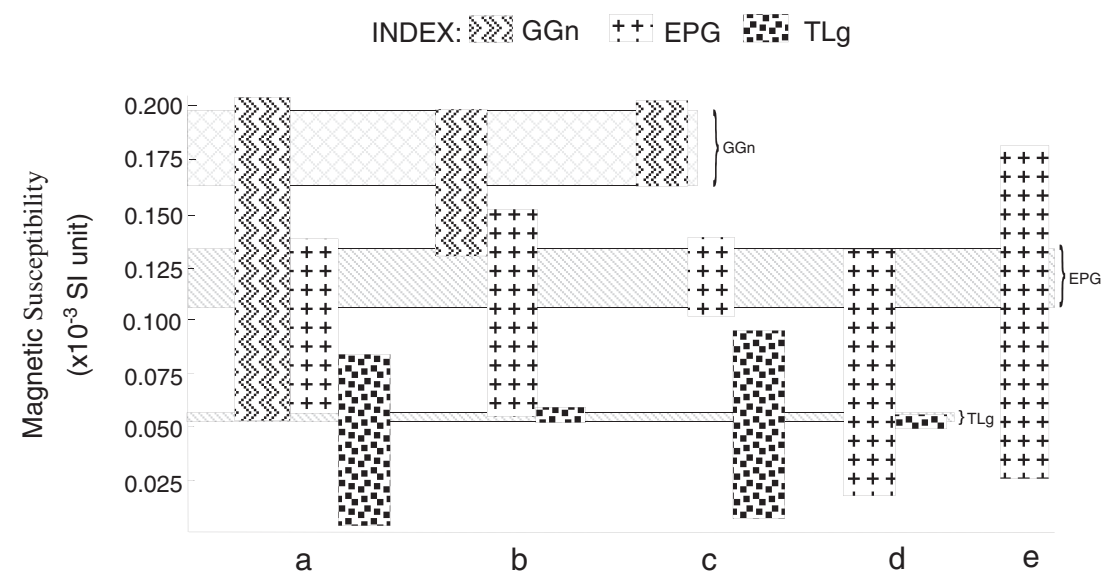

Figure 2. Bar diagram showing the variation range of magnetic susceptibility (MS) values $\left(\times 10^{-3}\right.$ SI unit) for GGn, EPG and TLg measured in the field along various transects. (a) In and around Ribba village, (b) Powari-Akpa transect, (c) Shongtong-Purbani transect, (d) Rakcham-Chitkul transect, and (e) Karcham-Rakcham transect. Horizontal bars show range of MS values for GGn, EPG and TLg common to all transects.

along their foliation and fracture planes. The TLg commonly contain small tourmaline crystals upto $2.5 \mathrm{~cm}$ long, but large size (6-10 cm long) fibrolitic tourmaline crystals together with mica flakes can also be found in a local pegmatite facies. The MS values of TLg range from 0.011 to $0.088 \times 10^{-3}$ SI with an average value of $0.049 \times 10^{-3}$ SI unit (figure 2).

\section{Analytical methods}

The modal mineralogy of representative samples of GGn, EPG and TLg has been determined using a James Swift automatic point counter. The magnetic susceptibility of granitoids was measured on smooth and fresh rock surfaces in the field using hand held SM-20 MS meter, which operated at $10 \mathrm{kHz}$ frequency with sensitivity $1 \times 10^{-6}$ SI unit. The instrument can operate in the temperature range of $10-60^{\circ} \mathrm{C}$. Measuring time of each reading is less than 3 seconds per sample in two steps. The MS value is corrected using recommended factors for degree of surface unevenness $(1 \mathrm{~mm}=$ $1.07,2 \mathrm{~mm}=1.15,3 \mathrm{~mm}=1.23,4 \mathrm{~mm}=1.32$, and $5 \mathrm{~mm}=1.41)$. Electron microprobe analysis of minerals was carried out at the Indian Institute of Technology-Roorkee, India using a JEOL JXA$8600 \mathrm{M}$, which was operated at $15 \mathrm{kV}$ accelerating voltage and $2 \times 10^{-8}$ A current. An electron beam diameter of $2 \mu \mathrm{m}$ was selected for most minerals, except feldspars for which a beam of $10 \mu \mathrm{m}$ diameter was preferred. An oxide ZAF correction procedure was employed. Whole rock major and trace elements ( $\mathrm{Ba}, \mathrm{Nb}, \mathrm{Rb}, \mathrm{Sr}, \mathrm{Y}$ and $\mathrm{Zr}$ ) were determined by XRF at Yamagata University, Japan, using fused glass beads and an XRF (Rigaku RIX 2000) with a Rh anode tube, operated at $50 \mathrm{kV}$ accelerating voltage, $50 \mathrm{~mA}$ current. The preparation of glass beads, calibration, matrix effect corrections and the precision of results using this methodology are described (Yamada et al. 1985; Murata 1993; Ban et al. 2005). Trace and rare earth elements (REE) were also analysed at the National Geophysical Research Institute, Hyderabad, India using ICP-MS (ELAN DRC II, Perkin-Elmer) optimised for maximum intensity $(\sim 40,000$ counts/sec) across the mass range and a $1 \mathrm{ng} / \mathrm{ml}$ solution of $\mathrm{Mg}$, $\mathrm{Rh}, \mathrm{In}, \mathrm{Ba}$, $\mathrm{Ce}, \mathrm{Pb}$ and U. Details of solution preparation, analytical methods, accuracy and precision of results are described (Balaram et al. 1996; Balaram and Gnaneshwar Rao 2003). Japanese rock reference materials (JG-2, JG-1a) were used to minimise the matrix effects.

\section{Petrography, modal mineralogy and mineral chemistry}

The granitic gneiss exhibits gneissose and hypidiomorphic to porphyritic textures and contains megacrysts (not porphyroblasts) of feldspars. A crude preferred orientation of flakes of biotite and muscovite gives rise to the gneissic appearance of the rocks. Some biotite and muscovite are bent suggesting deformation of ductile or non-ductile nature. Biotite is poikilitic with respect to plagioclase, K-feldspar and quartz. Plagioclase occurs as subhedral to euhedral laths, and is corroded at the margins due to replacement by perthitic $\mathrm{K}$-feldspars (orthoclase and microcline). Quartz is recrystallised because of low grade metamorphism. Apatite encloses zircon, and iron oxides occur as disseminated grains. The colour index $\left(\mathrm{M}^{\prime}\right)$ of GGn $\left(\mathrm{M}^{\prime}=6-12\right)$ ranges from hololeucocratic to 
leucocratic (LeMaitre 2002), and modal composition (table 1a) corresponds to Ms-Bt monzogranite and Ms-Bt granodiorite (figure 3). Representative analyses of plagioclase and K-feldspar from GGn are given in tables 2(a) and 3(a), respectively. Plagioclase from GGn $\left(\mathrm{An}_{10}\right.$ to $\left.\mathrm{An}_{31}\right)$ represents oligoclase to andesine (figure 4); however, rim composition is slightly more anorthitic compared to the core and this suggests reverse zoning. $\mathrm{K}$-feldspar in $\mathrm{GGn}\left(\mathrm{Or}_{88}-\mathrm{Or}_{95}\right)$ is mostly sanidine to orthoclase. It is likely that 10 micron beam size might have re-integrated the compositions of exsolution into K-feldspar. Representative analyses of biotite from GGn are given in table 4(a). Ferrous $\left(\mathrm{Fe}^{2+}\right)$ and ferric $\left(\mathrm{Fe}^{3+}\right)$ iron was obtained from total iron by partitioning following the stoichiometric methods of Dymek (1983) and Yavuz and Öztas (1997) for biotites from GGn, EPG and TLg. The biotite from GGn $\left(\mathrm{Mg} / \mathrm{Mg}+\mathrm{Fe}^{\mathrm{t}}=\right.$ 0.34-0.45) are classified as ferrobiotites enriched

Table 1(a). Modal mineral analysis (vol\%) and colour index $\left(M^{\prime}\right)$ of representative granite gneiss (GGn).

\begin{tabular}{|c|c|c|c|c|c|c|c|}
\hline Sample no. & C5 & G1 & G2 & G4 & $\mathrm{S} 2$ & $\mathrm{R} 1$ & $\mathrm{R} 4$ \\
\hline Quartz & 30.00 & 26.00 & 31.00 & 38.00 & 31.50 & 42.50 & 30.00 \\
\hline K-feldspar & 28.50 & 23.50 & 19.50 & 13.50 & 25.00 & 21.50 & 12.50 \\
\hline Plagioclase & 32.50 & 39.50 & 40.00 & 29.00 & 35.35 & 19.00 & 48.50 \\
\hline Biotite & 6.00 & 6.00 & 5.00 & 9.00 & 5.00 & 8.00 & 6.00 \\
\hline Muscovite & 2.00 & 4.00 & 2.50 & 7.50 & 2.00 & 6.50 & 2.00 \\
\hline Tourmaline & - & - & - & - & - & - & - \\
\hline Accessories & 1.00 & 1.00 & 1.00 & 2.00 & 1.00 & 2.50 & 1.00 \\
\hline Colour index $\left(\mathrm{M}^{\prime}\right)$ & 7.00 & 7.00 & 7.00 & 12.00 & 6.00 & 10.50 & 7.00 \\
\hline
\end{tabular}

Accessories include zircon (Zrn), apatite (Ap), opaques, etc. Colour index $\left(\mathrm{M}^{\prime}\right)=\mathrm{M}-$ light coloured minerals $(\mathrm{Ms}+\mathrm{Qtz}+$ Kfs $+\mathrm{Pl}$ ) after Streckeisen (1973), where M = 100. Following IUGS nomenclature (Streckeisen 1973; LeMaitre 2002) the GGn can be named as Ms-Bt monzogranite (sample nos. C5, G1, S2, R1) and Ms-Bt granodiorite (sample nos. G4, G2, R4). Mineral symbols are used after Kretz (1983).

Table 1(b). Modal mineral analysis (vol\%) and colour index ( $\left.M^{\prime}\right)$ of representative Early Palaeozoic granitoids (EPG).

\begin{tabular}{|c|c|c|c|c|c|c|c|c|c|c|c|c|c|c|c|c|}
\hline Sample no. & $\mathrm{C} 1$ & $\mathrm{C} 7$ & C9 & $\mathrm{C} 10$ & $\mathrm{C} 23$ & C19 & $\mathrm{C} 27$ & $\mathrm{~S} 1$ & $\mathrm{R} 2$ & R3 & $\mathrm{R} 6$ & A1 & A2 & A3 & A4 & A6 \\
\hline & 34.00 & 34.00 & 32.00 & 32.00 & 31.50 & 32.50 & 32.00 & 33.50 & 35.00 & 34.00 & & 33.50 & 34.00 & 30.00 & & 32.50 \\
\hline K-feldspar & 24.00 & 28.00 & 23.50 & 26.50 & 23.00 & 29.00 & 26.50 & 28.00 & 24.00 & 25.50 & 25.50 & 24.00 & 31.50 & 29.50 & 30.00 & 28.50 \\
\hline Plagioclase & 27.50 & 23.00 & 26.00 & 28.00 & 27.50 & 26.50 & 27.00 & 26.50 & 26.50 & 23.00 & 27.00 & 28.50 & 26.00 & 28.00 & 24.00 & 23.00 \\
\hline Biotite & 7.50 & 7.65 & 8.50 & 7.50 & 8.00 & 5.50 & 6.00 & 7.00 & 8.00 & 7.50 & 6.50 & 7.00 & 5. & 6.50 & 6. & 7.50 \\
\hline Muscovite & 4.00 & 4.25 & 4.50 & 3.00 & 4.50 & 2.50 & 4.50 & 3.00 & 4.50 & 5.50 & 4.50 & 5.00 & & 4.00 & 2.00 & 3.50 \\
\hline Tourmaline & 1.00 & - & - & - & - & 0.50 & - & - & - & 1.00 & - & - & 0.5 & - & - & - \\
\hline Accessories & 1.50 & 1.50 & 1.50 & 1.50 & & 1.00 & 2.00 & 2.00 & 1.00 & 2.50 & 1.00 & 1.0 & $1 .($ & 1.50 & 1.50 & 2.00 \\
\hline $\begin{array}{l}\text { Colour } \\
\text { index }\left(\mathrm{M}^{\prime}\right)\end{array}$ & 10.50 & 11.75 & 15.50 & 10.50 & 13.50 & 10.00 & 10.00 & 6.50 & 10.00 & 15.00 & 8.00 & 6.00 & 6.00 & 8.50 & 8.50 & 12.50 \\
\hline
\end{tabular}

Accessories include zircon (Zrn), apatite (Ap), opaques, etc. Colour index $\left(\mathrm{M}^{\prime}\right)=\mathrm{M}-$ light coloured minerals $(\mathrm{Ms}+\mathrm{Qtz}+$ $\mathrm{Kfs}+\mathrm{Pl}$ ) after Streckeisen (1973), where $\mathrm{M}=100$. Following IUGS nomenclature (Streckeisen 1973; LeMaitre 2002) all samples can be named as Ms-Bt leucomonzogranite. Mineral symbols are used after Kretz (1983).

Table 1(c). Modal mineral analysis (vol\%) and colour index ( $M^{\prime}$ ) of representative tourmaline bearing leucogranite (TLg).

\begin{tabular}{|c|c|c|c|c|c|c|c|c|}
\hline Sample no. & C6 & $\mathrm{C} 17 \mathrm{a}$ & $\mathrm{C} 17 \mathrm{~b}$ & A5 & TLg-2a & TLg-2b & TLg-2c & 2-TLg \\
\hline Quartz & 30.00 & 34.00 & 49.00 & 10.50 & 36.50 & 33.00 & 39.50 & 34.00 \\
\hline K-feldspar & 28.50 & 10.00 & 21.50 & 54.50 & 15.00 & 16.00 & 8.00 & 15.50 \\
\hline Plagioclase & 25.00 & 45.00 & 5.00 & 27.00 & 40.00 & 39.50 & 46.50 & 35.50 \\
\hline Biotite & 4.50 & 2.00 & 4.50 & 1.50 & 2.00 & 4.00 & 1.50 & 4.00 \\
\hline Muscovite & 7.00 & 4.50 & 8.50 & 3.50 & 5.00 & 5.00 & 3.00 & 6.50 \\
\hline Tourmaline & 3.00 & 2.50 & 4.00 & 3.00 & 2.00 & 1.50 & 1.00 & 2.50 \\
\hline Accessories & 1.00 & 1.00 & 3.50 & 0.50 & 1.00 & 1.00 & 1.00 & 1.00 \\
\hline Colour index $\left(\mathrm{M}^{\prime}\right)$ & 9.50 & 6.50 & 19.00 & 4.50 & 3.50 & 6.50 & 3.00 & 8.50 \\
\hline
\end{tabular}

Accessories include zircon (Zrn), apatite (Ap), opaques, etc. Colour index $\left(\mathrm{M}^{\prime}\right)=\mathrm{M}-$ light coloured minerals $(\mathrm{Ms}+\mathrm{Qtz}+$ $\mathrm{Kfs}+\mathrm{Pl}$ ) after Streckeisen (1973), where M = 100. Following IUGS nomenclature (Streckeisen 1973; LeMaitre 2002) the TLg can be named as Ms-Bt monzogranite (sample no. C6), Ms-Bt granodiorite (sample nos. C17a, 2 TLg, TLg-2a, TLg-2b, TLg-2c), Bt-Ms quartz rich granitoid (sample no. C17b) and Bt-Ms quartz syenite (sample no. A5). Mineral symbols are used after Kretz (1983). 


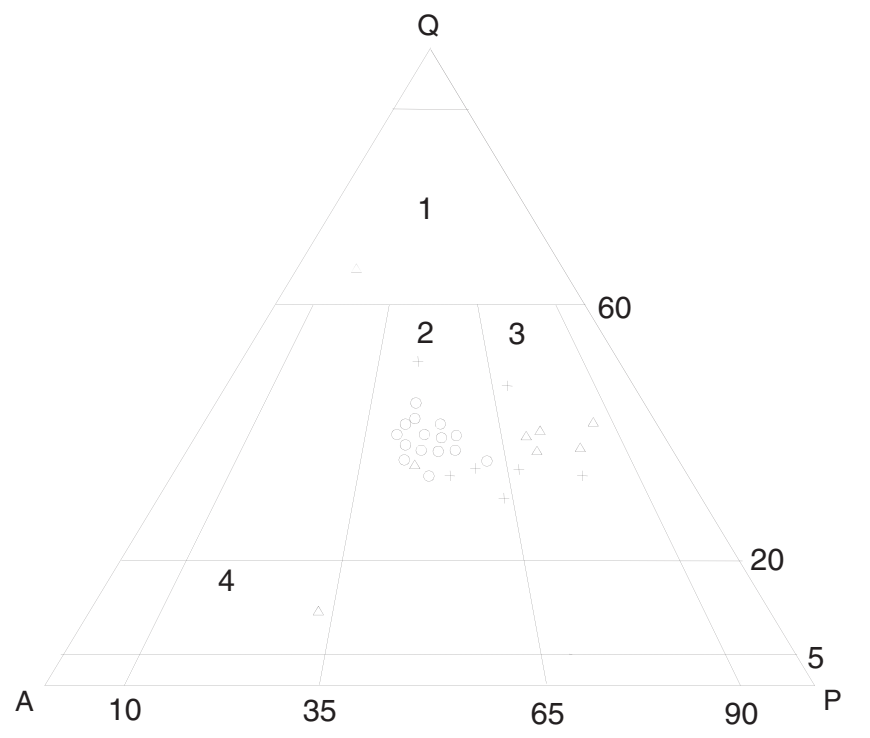

Figure 3. Modal compositions of EPG $(\bigcirc)$, GGn $(+)$ and TLg $(\Delta)$ in a $\mathrm{Q}-\mathrm{A}-\mathrm{P}$ diagram (LeMaitre 2002). $\mathrm{Q}=$ Quartz, $\mathrm{A}=$ Alkali feldspar, $\mathrm{P}=$ Plagioclase. Fields 1: quartz-rich granitoids, 2: monzogranite, 3: granodiorite, 4: quartz syenite.

in siderophyllite component and crystallised from a magma that was buffered mostly between FMQ and NNO (figure 5). The biotites show compositional affinity with biotite syncrystallised with muscovite (De Albuquerque 1973). The peraluminous (S-type) and reduced nature of GGn melt is clearly indicated by the dominant $3 \mathrm{Fe} \rightleftharpoons 2 \mathrm{Al}$ and $3 \mathrm{Mg} \rightleftharpoons 2 \mathrm{Al}$ substitutions in the biotite (figure $6 \mathrm{a}$, b) and by the large variations in the $\mathrm{Fe}^{\mathrm{t}} / \mathrm{Fe}^{\mathrm{t}}+\mathrm{Mg}$ ratios $(\mathrm{GGn}=0.55-0.66)$ during the evolution of the biotite from their respective felsic melts. Representative analyses of muscovite from GGn and their empirically estimated Li-contents (Tindle and Webb 1990; Tischendorf 1997; Tischendorf et al. 1999; Yavuz 2001) are given in table 5(a). Muscovite in GGn $\left(\mathrm{Mg} / \mathrm{Mg}+\mathrm{Fe}^{\mathrm{t}}=0.58-0.66\right)$ represent celadonitic and paragonitic solid-solutions (Miller et al. 1981) and belong to the $\mathrm{Li}-\mathrm{Al}$ mica group; more precisely they represent lithian muscovite, lepidolite and zinnwaldite (figure 7), which were most likely crystallised in a peraluminous felsic melt that underwent differentiation. The substitution of ferro-celadonite in muscovite of GGn, EPG and TLg involves the octahedral sites where $\mathrm{Al}^{3+}$ is mainly substituted for by $\mathrm{Fe}, \mathrm{Mg}^{2+}$ and minor amounts of $\mathrm{Ti}$ and $\mathrm{Mn}^{2+}$ because of the low $\mathrm{Ti}^{4+}$ content of the mica. The compositional gaps in the data set could indicate a geochemical hiatus caused either by inadequate sampling or may be geologic in nature.

The EPG exhibit fine- to coarse-grained, equigranular to inequigranular, hypidiomorphic textures and are composed of plagioclase, biotite, muscovite, K-feldspar, quartz, tourmaline, apatite and zircon. Scattered iron oxides are present. Plagioclase $(3-10 \mathrm{~mm})$ occurs as subhedral to euhedral lath-shaped grains, which are intergrown with quartz to produce myrmekite. Primary biotites show sub-poikilitic to poikilitic relations with magnetite, apatite, zircon, monazite, and plagioclase. Randomly oriented crystals of muscovite exhibit an interlocking relationship with the primary biotite. Microcline contains inclusions of plagioclase indicating growth of perthite, whereas orthoclase displays simple twinning. Quartz that occurs as an interstitial phase contains inclusions of apatite, zircon, and tourmaline. Tourmaline also occurs in microveinlets. The colour index $\left(\mathrm{M}^{\prime}\right)$ of EPG $\left(\mathrm{M}^{\prime}=6-12\right)$ ranges from hololeucocratic to leucocratic (LeMaitre 2002), and modal composition (table 1b) corresponds to Ms-Bt monzogranite (figure 3). Representative analyses of plagioclase and K-feldspar from EPG are given in tables 2(b) and $3(\mathrm{~b})$, respectively. Plagioclase from EPG $\left(\mathrm{An}_{15}-\mathrm{An}_{33}\right)$ represents oligoclase to andesine composition (figure 4). Reverse zoning is common in plagioclase of EPG. K-feldspar in EPG $\left(\mathrm{Or}_{86^{-}}\right.$ $\left.\mathrm{Or}_{97}\right)$ is mostly sanidine to orthoclase. Representative analyses of biotite from EPG are given in table $4(\mathrm{~b})$. The biotite from EPG $\left(\mathrm{Mg} / \mathrm{Mg}+\mathrm{Fe}^{\mathrm{t}}=\right.$ 0.27-0.47) represents ferrobiotites enriched in siderophyllite component buffered mostly between FMQ and NNO (figure 5). EPG biotites show compositional affinity with biotite syncrystallised with muscovite (De Albuquerque 1973). Dominant $3 \mathrm{Fe} \rightleftharpoons 2 \mathrm{Al}$ and $3 \mathrm{Mg} \rightleftharpoons 2 \mathrm{Al}$ substitutions in $\mathrm{EPG}$ biotite (figure $6 \mathrm{a}, \mathrm{b}$ ) and large variations in the $\mathrm{Fe}^{\mathrm{t}} / \mathrm{Fe}^{\mathrm{t}}+\mathrm{Mg}$ ratios $(\mathrm{EPG}=0.53-0.73)$ suggests its peraluminous (S-type) and reduced nature. The observed large variation in the $\mathrm{Fe}^{\mathrm{t}} / \mathrm{Fe}^{\mathrm{t}}+\mathrm{Mg}$ ratio of biotites from EPG and TLg should have been primarily controlled by changing the activity of silica $\left(a_{\mathrm{SiO}_{2}}\right)$ of the host magmas during fractional differentiation. Representative analyses of muscovites from EPG and empirically estimated Li-contents (Tindle and Webb 1990; Tischendorf 1997; Tischendorf et al. 1999; Yavuz 2001) are given in table $5(\mathrm{~b})$. Muscovite in $\mathrm{EPG}\left(\mathrm{Mg} / \mathrm{Mg}+\mathrm{Fe}^{\mathrm{t}}=\right.$ 0.31-0.59) represent celadonitic and paragonitic solid-solutions (Miller et al. 1981), belonging to the Li-Al mica (lithian muscovite, lepidolite and zinnwaldite, figure 7). Representative analyses of tourmaline from EPG is given in table 6(a). $\mathrm{Fe}^{3+}$ and $\mathrm{Fe}^{2+}$ were obtained from total iron by partition using the tourmal program (Yavuz 1997). It has been assumed that boron has a stoichiometric abundance of 3 apfu (e.g., Torres-Ruiz et al. 2003). Tourmaline from EPG belong principally to the vacancy and alkali groups (Hawthorne and Henry 1999) and contain minor amounts of Mn (tsiliasite component; Slivko 1959), but lack 
Table 2. Representative electron microprobe analysis of plagioclase from (a) granite gneiss (GGn), (b) Early Palaeozoic granitoids (EPG), and (c) Tourmaline bearing leucogranite (TLg).

\begin{tabular}{|c|c|c|c|}
\hline & Range & Mean & Std. dev. \\
\hline \multicolumn{4}{|c|}{ (a) 18 samples Pl (GGn) } \\
\hline $\mathrm{SiO}_{2}$ & $59.45-65.82$ & 61.76 & 1.87 \\
\hline $\mathrm{Al}_{2} \mathrm{O}_{3}$ & $21.11-25.47$ & 24.29 & 1.16 \\
\hline $\mathrm{CaO}$ & $2.04-6.35$ & 5.48 & 1.33 \\
\hline $\mathrm{Na}_{2} \mathrm{O}$ & $7.68-9.87$ & 8.64 & 0.64 \\
\hline $\mathrm{K}_{2} \mathrm{O}$ & $0.09-0.57$ & 0.33 & 0.12 \\
\hline $\mathrm{FeO}$ & $0.00-0.1$ & 0.02 & 0.02 \\
\hline \multicolumn{4}{|c|}{ Structural formula based on 8 oxygen basis } \\
\hline An $(\%)$ & $10.20-30.80$ & 25.42 & 5.95 \\
\hline $\mathrm{Ab}(\%)$ & $67.30-89.30$ & 72.81 & 6.37 \\
\hline Or $(\%)$ & $0.50-3.20$ & 1.77 & 0.67 \\
\hline \multicolumn{4}{|c|}{ (b) 17 samples Pl (EPG) } \\
\hline $\mathrm{SiO}_{2}$ & $59.58-65.82$ & 63.27 & 2.21 \\
\hline $\mathrm{Al}_{2} \mathrm{O}_{3}$ & $21.16-26.38$ & 23.72 & 1.55 \\
\hline $\mathrm{CaO}$ & $3.01-6.65$ & 4.35 & 1.44 \\
\hline $\mathrm{Na}_{2} \mathrm{O}$ & $6.83-10.07$ & 8.77 & 0.93 \\
\hline $\mathrm{K}_{2} \mathrm{O}$ & $0.11-0.96$ & 0.45 & 0.31 \\
\hline $\mathrm{FeO}$ & $0.00-0.05$ & 0.01 & 0.02 \\
\hline \multicolumn{4}{|c|}{ Structural formula based on 8 oxygen basis } \\
\hline An $(\%)$ & $14.50-33.20$ & 21.09 & 7.15 \\
\hline $\mathrm{Ab}(\%)$ & $65.80-84.30$ & 76.46 & 6.26 \\
\hline Or $(\%)$ & $0.60-5.10$ & 2.46 & 1.70 \\
\hline \multicolumn{4}{|c|}{ (c) 26 samples Pl (TLg) } \\
\hline $\mathrm{SiO}_{2}$ & $63.64-69.04$ & 66.09 & 1.60 \\
\hline $\mathrm{Al}_{2} \mathrm{O}_{3}$ & $25.69-29.31$ & 22.29 & 1.15 \\
\hline $\mathrm{CaO}$ & $0.62-3.11$ & 2.23 & 0.78 \\
\hline $\mathrm{Na}_{2} \mathrm{O}$ & $7.33-12.48$ & 10.26 & 1.10 \\
\hline $\mathrm{K}_{2} \mathrm{O}$ & $0.08-0.36$ & 0.20 & 0.10 \\
\hline $\mathrm{FeO}$ & $0.00-0.04$ & 0.01 & 0.01 \\
\hline \multicolumn{4}{|c|}{ Structural formula based on 8 oxygen basis } \\
\hline An $(\%)$ & $2.80-15.10$ & 10.74 & 3.79 \\
\hline $\mathrm{Ab}(\%)$ & $83.20-96.70$ & 88.4 & 4.05 \\
\hline Or $(\%)$ & $0.50-2.20$ & 1.14 & 0.54 \\
\hline
\end{tabular}

Note: The entire database can be obtained from the corresponding author.

absolutely any empirically estimated $\mathrm{Fe}^{3+}$ content, therefore, demonstrating an affinity with schorlelbaite group (figure 8). In order to saturate vacant sites ( $<6$ apfu), some Al substitute for $\mathrm{Si}$ at Tsite (Foit and Rosenberg 1979) and Mg at Z-site (e.g., Torres-Ruiz et al. 2003) in the tourmalines from EPG and TLg. The $\mathrm{Fe} /(\mathrm{Fe}+\mathrm{Mg})$ ratio increases with $\mathrm{Al}$ substitution $\left[(\mathrm{Mg}, \mathrm{Fe})_{-1} \mathrm{Si}_{-1} \mathrm{Al}_{2}\right]$ in the Y-site (London and Manning 1995) and, therefore, samples plot in the field below the line $\Sigma(\mathrm{Fe}+\mathrm{Mg})<3$. In terms of $\mathrm{Al}-\mathrm{Fe}^{\mathrm{t}}-\mathrm{Mg}$ (Henry and Guidotti 1985) the tourmaline from EPG and TLg appear to have crystallised from a lithium-poor, tur-bt granitic melt or its derivative pegmatites and aplites (figure 9).
Table 3. Representative electron microprobe analysis of $K$ feldspar from (a) granite gneiss (GGn), (b) Early Palaeozoic granitoids (EPG), and (c) tourmaline bearing leucogranite (TLg).

\begin{tabular}{|c|c|c|c|}
\hline & Range & Mean & Std. dev. \\
\hline \multicolumn{4}{|c|}{ (a) 4 samples Kfs (GGn) } \\
\hline $\mathrm{SiO}_{2}$ & $63.99-65.01$ & 64.41 & 0.43 \\
\hline $\mathrm{Al}_{2} \mathrm{O}_{3}$ & $17.93-18.75$ & 18.49 & 0.38 \\
\hline $\mathrm{CaO}$ & $0.02-0.08$ & 0.04 & 0.03 \\
\hline $\mathrm{Na}_{2} \mathrm{O}$ & $0.55-1.40$ & 1.13 & 0.39 \\
\hline $\mathrm{K}_{2} \mathrm{O}$ & $14.94-15.55$ & 15.29 & 0.30 \\
\hline $\mathrm{FeO}$ & $0.00-0.02$ & 0.01 & 0.01 \\
\hline \multicolumn{4}{|c|}{ Structural formula based on 8 oxygen basis } \\
\hline An $(\%)$ & $0.10-0.40$ & 0.23 & 0.13 \\
\hline $\mathrm{Ab}(\%)$ & $5.00-12.30$ & 9.95 & 3.35 \\
\hline Or $(\%)$ & $87.50-94.90$ & 89.83 & 3.43 \\
\hline \multicolumn{4}{|c|}{ (b) 7 samples Kfs (EPG) } \\
\hline $\mathrm{SiO}_{2}$ & $46.26-65.60$ & 61.73 & 6.94 \\
\hline $\mathrm{Al}_{2} \mathrm{O}_{3}$ & $18.25-33.12$ & 20.99 & 5.37 \\
\hline $\mathrm{CaO}$ & $0.00-0.04$ & 0.02 & 0.01 \\
\hline $\mathrm{Na}_{2} \mathrm{O}$ & $0.11-1.68$ & 0.80 & 0.61 \\
\hline $\mathrm{K}_{2} \mathrm{O}$ & $10.44-21.62$ & 16.52 & 3.69 \\
\hline $\mathrm{FeO}$ & $0.00-2.00$ & 0.29 & 0.75 \\
\hline \multicolumn{4}{|c|}{ Structural formula based on 8 oxygen basis } \\
\hline An $(\%)$ & $0.00-0.20$ & 0.09 & 0.07 \\
\hline $\mathrm{Ab}(\%)$ & $1.00-13.50$ & 6.64 & 4.72 \\
\hline Or $(\%)$ & $85.60-98.90$ & 93.27 & 4.71 \\
\hline \multicolumn{4}{|c|}{ (c) 7 samples Kfs (TLg) } \\
\hline $\mathrm{SiO}_{2}$ & $63.33-66.09$ & 64.98 & 1.08 \\
\hline $\mathrm{Al}_{2} \mathrm{O}_{3}$ & $18.28-19.33$ & 18.66 & 0.38 \\
\hline $\mathrm{CaO}$ & $0.01-0.04$ & 0.02 & 0.01 \\
\hline $\mathrm{Na}_{2} \mathrm{O}$ & $0.66-1.44$ & 1.08 & 0.33 \\
\hline $\mathrm{K}_{2} \mathrm{O}$ & $14.88-20.88$ & 17.21 & 2.88 \\
\hline $\mathrm{FeO}$ & $0.00-0.02$ & 0.01 & 0.01 \\
\hline \multicolumn{4}{|c|}{ Structural formula based on 8 oxygen basis } \\
\hline An $(\%)$ & $0.00-0.10$ & 0.08 & 0.04 \\
\hline $\mathrm{Ab}(\%)$ & $5.80-12.90$ & 8.87 & 3.06 \\
\hline Or $(\%)$ & $87.00-94.10$ & 91.05 & 3.07 \\
\hline
\end{tabular}

Note: The entire database can be obtained from the corresponding author.

The TLg is medium to coarse grained (1-10 mm) and exhibits perthite, myrmekite and graphic integrowths. Plagioclase is partly altered to sericite, and poikilitically encloses mica. Primary biotite and muscovite occur as randomly oriented crystals. K-feldspar occurs as perthite microcline and orthoclase, which commonly enclose quartz, biotite and muscovite. Quartz is interstitial. Tourmaline occurs as zoned crystals. Apatite and zircon are included in the biotite, muscovite and feldspars, and iron oxides occur as disseminated grains. The colour index $\left(\mathrm{M}^{\prime}\right)$ of $\mathrm{TLg}\left(\mathrm{M}^{\prime}=3-19\right)$ ranges from hololeucocratic to leucocratic (LeMaitre 2002), and modal composition (table 1c) corresponds to $\mathrm{Bt}-\mathrm{Ms}$ monzogranite, Bt-Ms granodiorite, Bt-Ms 


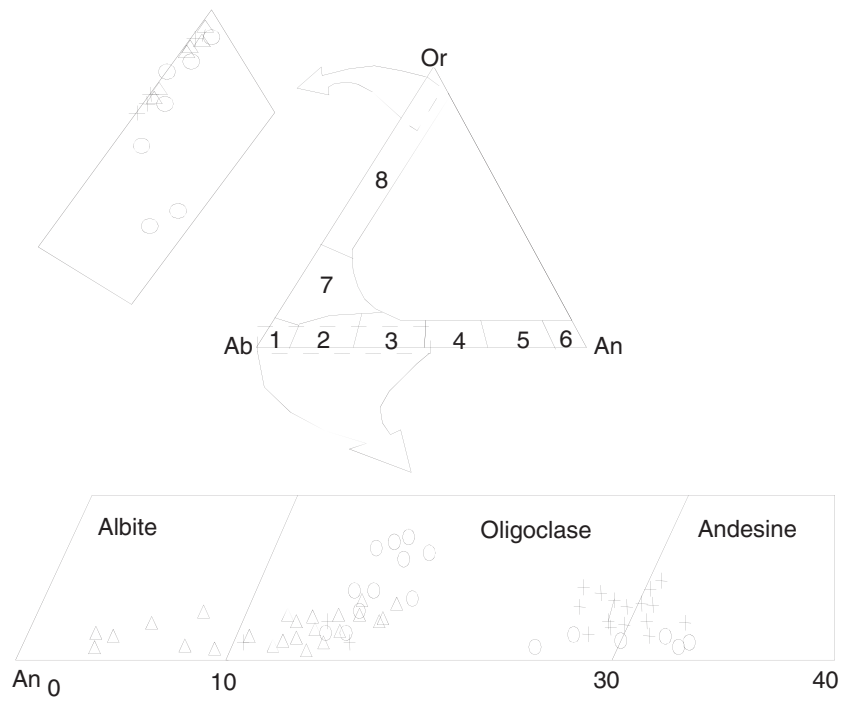

Figure 4. Albite-Orthoclase-Anorthite diagram showing plagioclase and K-feldspar compositions from GGn $(+)$, EPG $(\bigcirc)$ and TLg $(\Delta)$ samples. Fields; 1: albite, 2: oligoclase, 3: andesine, 4: labradorite, 5: bytonite, 6: anorthite, 7: anorthoclase, and 8: sanidine.

Table 4. Representative electron microprobe analysis of biotite from (a) granite gneiss (GGn), (b) Early Palaeozoic granitoids (EPG), and (c) tourmaline bearing leucogranite (TLg).

\begin{tabular}{|c|c|c|c|}
\hline & Range & Mean & Std. dev. \\
\hline \multicolumn{4}{|c|}{ (a) 18 samples Bt (GGn) } \\
\hline $\mathrm{SiO}_{2}$ & $32.71-37.38$ & 34.85 & 1.00 \\
\hline $\mathrm{Al}_{2} \mathrm{O}_{3}$ & $17.64-20.82$ & 18.73 & 0.78 \\
\hline $\mathrm{TiO}_{2}$ & $4.05-5.34$ & 4.59 & 0.38 \\
\hline $\mathrm{FeO}$ & $17.26-23.82$ & 20.09 & 1.30 \\
\hline $\mathrm{MnO}$ & $0.00-0.11$ & 0.05 & 0.03 \\
\hline $\mathrm{MgO}$ & $5.04-8.95$ & 8.01 & 0.85 \\
\hline $\mathrm{CaO}$ & $0.00-0.07$ & 0.01 & 0.02 \\
\hline $\mathrm{Na}_{2} \mathrm{O}$ & $0.00-0.32$ & 0.16 & 0.10 \\
\hline $\mathrm{K}_{2} \mathrm{O}$ & $8.97-9.97$ & 9.55 & 0.25 \\
\hline \multicolumn{4}{|c|}{ Structural formula based on 11 oxygen basis } \\
\hline $\mathrm{Si}$ & $2.53-2.81$ & 2.65 & 0.05 \\
\hline $\mathrm{Al}^{\mathrm{IV}}$ & $1.19-1.47$ & 1.35 & 0.05 \\
\hline$\Sigma Z=4$ & 4.00 & 4.00 & 0.00 \\
\hline $\mathrm{Al}^{\mathrm{VI}}$ & $0.13-0.65$ & 0.33 & 0.11 \\
\hline $\mathrm{Ti}$ & $0.23-0.31$ & 0.26 & 0.02 \\
\hline $\mathrm{Fe}^{3+}$ & $0.02-0.68$ & 0.22 & 0.18 \\
\hline $\mathrm{Fe}^{2+}$ & $0.42-1.24$ & 1.06 & 0.18 \\
\hline Mn & $0.00-0.01$ & 0.00 & 0.00 \\
\hline $\mathrm{Mg}$ & $0.56-1.02$ & 0.91 & 0.10 \\
\hline$\Sigma Y \sim 3$ & $2.53-2.91$ & 2.78 & 0.08 \\
\hline $\mathrm{Ca}$ & $0.00-0.01$ & 0.00 & 0.00 \\
\hline $\mathrm{Na}$ & $0.00-0.05$ & 0.02 & 0.01 \\
\hline K & $0.8-0.96$ & 0.92 & 0.03 \\
\hline$\Sigma X \sim 1$ & $0.80-1.00$ & 0.95 & 0.04 \\
\hline $\mathrm{Fe}^{\#}$ & $0.55-0.66$ & 0.59 & 0.03 \\
\hline $\mathrm{Mg} \#$ & $0.34-0.45$ & 0.41 & 0.03 \\
\hline \multicolumn{4}{|c|}{ (b) 47 samples Bt (EPG) } \\
\hline $\mathrm{SiO}_{2}$ & $32.30-36.10$ & 33.90 & 0.84 \\
\hline $\mathrm{Al}_{2} \mathrm{O}_{3}$ & $13.78-19.25$ & 16.75 & 1.11 \\
\hline
\end{tabular}

Table 4. (Continued.)

\begin{tabular}{|c|c|c|c|}
\hline & Range & Mean & Std. dev. \\
\hline $\mathrm{TiO}_{2}$ & $2.10-3.95$ & 3.36 & 0.35 \\
\hline $\mathrm{FeO}$ & $19.52-24.44$ & 22.61 & 1.01 \\
\hline $\mathrm{MnO}$ & $0.01-0.62$ & 0.35 & 0.11 \\
\hline $\mathrm{MgO}$ & $5.01-7.26$ & 6.44 & 0.59 \\
\hline $\mathrm{CaO}$ & $0.00-0.14$ & 0.03 & 0.04 \\
\hline $\mathrm{Na}_{2} \mathrm{O}$ & $0.00-0.56$ & 0.07 & 0.10 \\
\hline $\mathrm{K}_{2} \mathrm{O}$ & $9.22-13.41$ & 12.09 & 1.02 \\
\hline \multicolumn{4}{|c|}{ Structural formula based on 11 oxygen basis } \\
\hline $\mathrm{Si}$ & $2.60-2.78$ & 2.68 & 0.04 \\
\hline $\mathrm{Al}^{\mathrm{IV}}$ & $1.22-1.40$ & 1.32 & 0.04 \\
\hline$\Sigma Z=4$ & 4.00 & 4.00 & 0.00 \\
\hline $\mathrm{Al}^{\mathrm{VI}}$ & $0.11-0.47$ & 0.24 & 0.08 \\
\hline $\mathrm{Ti}$ & $0.16-0.23$ & 0.20 & 0.02 \\
\hline $\mathrm{Fe}^{3+}$ & $0.11-0.50$ & 0.24 & 0.09 \\
\hline $\mathrm{Fe}^{2+}$ & $0.95-1.46$ & 1.25 & 0.12 \\
\hline $\mathrm{Mn}$ & $0.00-0.04$ & 0.02 & 0.01 \\
\hline $\mathrm{Mg}$ & $0.60-0.86$ & 0.76 & 0.06 \\
\hline$\Sigma Y \sim 3$ & $2.62-2.82$ & 2.72 & 0.04 \\
\hline $\mathrm{Ca}$ & $0.00-0.01$ & 0.00 & 0.00 \\
\hline $\mathrm{Na}$ & $0.00-0.09$ & 0.01 & 0.02 \\
\hline K & $0.93-1.39$ & 1.22 & 0.11 \\
\hline$\Sigma \mathrm{X} \sim 1$ & $0.95-1.39$ & 1.23 & 0.11 \\
\hline $\mathrm{Fe}^{\#}$ & $0.53-0.73$ & 0.66 & 0.03 \\
\hline $\mathrm{Mg} \#$ & $0.27-0.47$ & 0.34 & 0.03 \\
\hline \multicolumn{4}{|c|}{ (c) 09 samples Bt (TLg) } \\
\hline $\mathrm{SiO}_{2}$ & $33.35-36.38$ & 34.41 & 1.06 \\
\hline $\mathrm{Al}_{2} \mathrm{O}_{3}$ & $18.53-20.61$ & 19.55 & 0.74 \\
\hline $\mathrm{TiO}_{2}$ & $1.61-2.05$ & 1.87 & 0.14 \\
\hline $\mathrm{FeO}$ & $22.95-24.89$ & 24.12 & 0.59 \\
\hline $\mathrm{MnO}$ & $0.37-0.71$ & 0.50 & 0.13 \\
\hline $\mathrm{MgO}$ & $4.49-6.01$ & 5.15 & 0.58 \\
\hline $\mathrm{CaO}$ & $0.00-0.06$ & 0.03 & 0.02 \\
\hline $\mathrm{Na}_{2} \mathrm{O}$ & $0.00-0.18$ & 0.05 & 0.07 \\
\hline $\mathrm{K}_{2} \mathrm{O}$ & $9.35-9.85$ & 9.64 & 0.17 \\
\hline \multicolumn{4}{|c|}{ Structural formula based on 11 oxygen basis } \\
\hline $\mathrm{Si}$ & $2.64-2.75$ & 2.69 & 0.04 \\
\hline $\mathrm{Al}^{\mathrm{IV}}$ & $1.25-1.36$ & 1.31 & 0.04 \\
\hline$\Sigma Z=4$ & 4.00 & 4.00 & 0.00 \\
\hline $\mathrm{Al}^{\mathrm{VI}}$ & $0.40-0.58$ & 0.49 & 0.07 \\
\hline $\mathrm{Ti}$ & $0.09-0.12$ & 0.11 & 0.01 \\
\hline $\mathrm{Fe}^{3+}$ & $0.05-0.43$ & 0.23 & 0.15 \\
\hline $\mathrm{Fe}^{2+}$ & $1.09-1.58$ & 1.34 & 0.19 \\
\hline Mn & $0.02-0.05$ & 0.04 & 0.01 \\
\hline $\mathrm{Mg}$ & $0.52-0.70$ & 0.60 & 0.07 \\
\hline$\Sigma Y \sim 3$ & $2.76-2.88$ & 2.81 & 0.05 \\
\hline $\mathrm{Ca}$ & 0.00 & 0.00 & 0.00 \\
\hline $\mathrm{Na}$ & $0.00-0.03$ & 0.01 & 0.01 \\
\hline K & $0.93-0.98$ & 0.96 & 0.02 \\
\hline$\Sigma X \sim 1$ & $0.94-0.99$ & 0.97 & 0.02 \\
\hline $\mathrm{Fe}^{\#}$ & $0.70-0.75$ & 0.73 & 0.02 \\
\hline $\mathrm{Mg}^{\#}$ & $0.25-0.30$ & 0.27 & 0.02 \\
\hline
\end{tabular}

$\mathrm{Fe}^{\#}=\mathrm{Fe}^{\mathrm{t}} / \mathrm{Fe}^{\mathrm{t}}+\mathrm{Mg} ; \mathrm{Mg}^{\#}=\mathrm{Mg} / \mathrm{Mg}+\mathrm{Fe}^{\mathrm{t}}$.

Note: The entire database can be obtained from the corresponding author. 


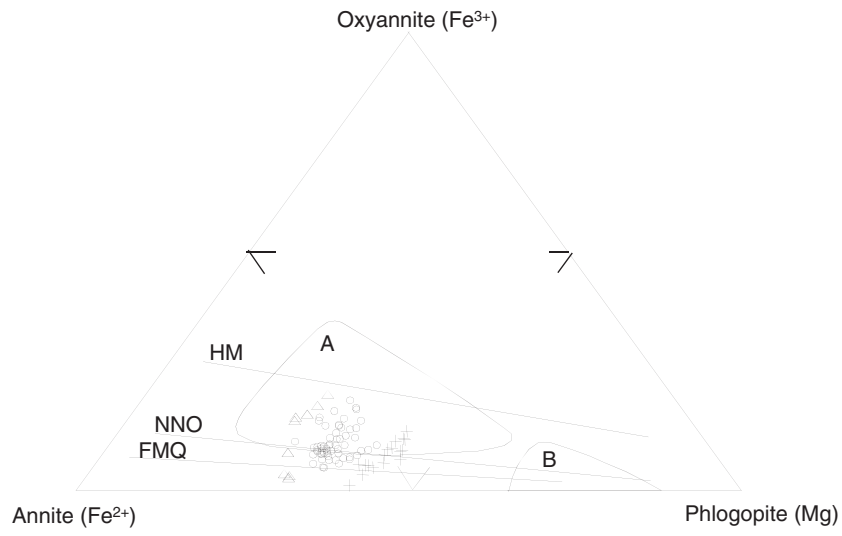

Figure 5. Annite--oxyannite-phlogopite plot for biotite showing (A) primary (Foster 1960) and (B) hydrothermal (Beane 1974) biotite fields. The HM, NNO and FMQ buffer curves are taken after Wones and Eugster (1965). Most EPG $(\bigcirc)$ biotites show slightly different trend as compared to GGn $(+)$ and $\operatorname{TLg}(\Delta)$ biotites.

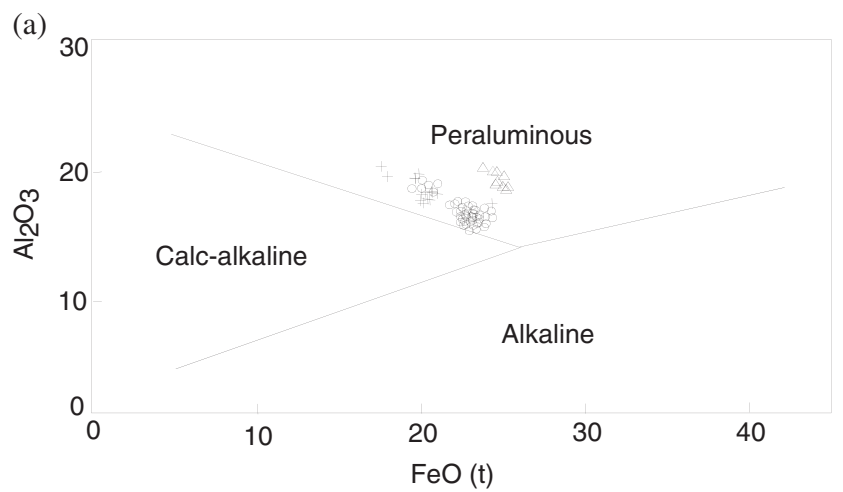

(b)

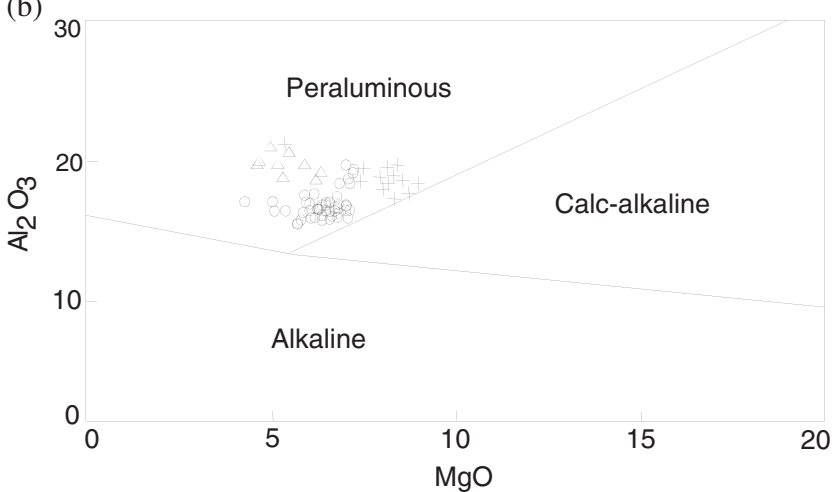

Figure 6. (a) $\mathrm{Al}_{2} \mathrm{O}_{3}$ vs. $\mathrm{FeO}(\mathrm{t})$ plot for biotite from GGn $(+)$, EPG $(\bigcirc)$ and TLg $(\Delta)$. The fields of biotite that crystallised in anorogenic alkaline, calc-alkaline orogenic suites and peraluminous (including S-types) magmas are shown, after Abdel-Rahman (1994). (b) $\mathrm{Al}_{2} \mathrm{O}_{3}$ vs. $\mathrm{MgO}$ plot for biotite from GGn $(+), \operatorname{EPG}(\mathrm{o})$ and $\operatorname{TLg}(\Delta)$. The fields of biotite that crystallised in anorogenic alkaline, calc-alkaline orogenic suites and peraluminous (including S-types) magmas are shown after Abdel-Rahman (1994).

quartz rich granitoid and Bt-Ms quartz syenite (figure 3).

Representative analyses of plagioclase and Kfeldspar from TLg are given in tables 2(c) and 3(c),
Table 5. Representative electron microprobe analysis of muscovite from (a) granite gneiss (GGn), (b) Early Palaeozoic granitoids (EPG), and (c) tourmaline bearing leucogranite (TLg).

\begin{tabular}{|c|c|c|c|}
\hline & Range & Mean & Std. dev. \\
\hline \multicolumn{4}{|c|}{ (a) 10 samples Ms (GGn) } \\
\hline $\mathrm{SiO}_{2}$ & $49.61-51.36$ & 50.64 & 0.58 \\
\hline $\mathrm{TiO}_{2}$ & $1.76-2.08$ & 1.95 & 0.11 \\
\hline $\mathrm{Al}_{2} \mathrm{O}_{3}$ & $35.62-37.09$ & 36.46 & 0.45 \\
\hline $\mathrm{FeO}$ & $0.99-1.31$ & 1.12 & 0.11 \\
\hline $\mathrm{MnO}$ & $0.00-0.03$ & 0.01 & 0.01 \\
\hline $\mathrm{MgO}$ & $0.78-1.22$ & 0.92 & 0.13 \\
\hline $\mathrm{CaO}$ & $0.00-0.03$ & 0.01 & 0.01 \\
\hline $\mathrm{Na}_{2} \mathrm{O}$ & $0.00-0.18$ & 0.10 & 0.06 \\
\hline $\mathrm{K}_{2} \mathrm{O}$ & $4.22-5.10$ & 4.72 & 0.26 \\
\hline $\mathrm{Li}_{2} \mathrm{O}(\mathrm{c})$ & $4.69-5.19$ & 4.98 & 0.16 \\
\hline \multicolumn{4}{|c|}{ Structural formula based on 22 oxygen basis } \\
\hline $\mathrm{Si}$ & $3.00-3.04$ & 3.03 & 0.01 \\
\hline $\mathrm{Al}^{\mathrm{IV}}$ & $0.96-1.00$ & 0.97 & 0.01 \\
\hline$\Sigma Z=4$ & 4 & 4.00 & 0.00 \\
\hline${ }^{\mathrm{VI}} \mathrm{Al}$ & $1.56-1.62$ & 1.60 & 0.02 \\
\hline $\mathrm{VI}_{\mathrm{Ti}}$ & $0.08-0.10$ & 0.09 & 0.01 \\
\hline${ }^{\mathrm{VI}} \mathrm{Fe}^{2+}$ & $0.05-0.07$ & 0.06 & 0.01 \\
\hline${ }^{\mathrm{VI}} \mathrm{Mn}$ & $0.00-0.00$ & 0.00 & 0.00 \\
\hline${ }^{\mathrm{VI}} \mathrm{Mg}$ & $0.07-0.11$ & 0.08 & 0.01 \\
\hline $\mathrm{Li}$ & $1.15-2.16$ & 1.30 & 0.30 \\
\hline$\Sigma \mathrm{Y} \sim 3$ & 3 & 3.12 & 0.31 \\
\hline $\mathrm{Ca}$ & $0.00-0.00$ & 0.00 & 0.00 \\
\hline $\mathrm{Na}$ & $0.00-0.02$ & 0.01 & 0.01 \\
\hline K & $0.32-0.39$ & 0.36 & 0.02 \\
\hline$\Sigma \mathrm{X} \sim 1$ & $0.34-0.40$ & 0.38 & 0.02 \\
\hline $\mathrm{Fe}^{\#}$ & $0.35-0.44$ & 0.40 & 0.03 \\
\hline $\mathrm{Mg}^{\#}$ & $0.58-0.66$ & 0.62 & 0.03 \\
\hline \multicolumn{4}{|c|}{ (b) 17 samples Ms (EPG) } \\
\hline $\mathrm{SiO}_{2}$ & $49.16-52.90$ & 50.38 & 0.96 \\
\hline $\mathrm{TiO}_{2}$ & $0.02-1.05$ & 0.57 & 0.29 \\
\hline $\mathrm{Al}_{2} \mathrm{O}_{3}$ & $32.20-37.25$ & 35.15 & 1.76 \\
\hline $\mathrm{FeO}$ & $1.31-2.13$ & 1.68 & 0.24 \\
\hline $\mathrm{MnO}$ & $0.00-0.06$ & 0.02 & 0.02 \\
\hline $\mathrm{MgO}$ & $0.46-1.65$ & 0.99 & 0.29 \\
\hline $\mathrm{CaO}$ & $0.00-0.04$ & 0.02 & 0.01 \\
\hline $\mathrm{Na}_{2} \mathrm{O}$ & $0.00-0.42$ & 0.22 & 0.13 \\
\hline $\mathrm{K}_{2} \mathrm{O}$ & $4.92-8.70$ & 6.02 & 1.00 \\
\hline $\mathrm{Li}_{2} \mathrm{O}(\mathrm{c})$ & $4.56-5.63$ & 4.91 & 0.28 \\
\hline \multicolumn{4}{|c|}{ Structural formula based on 22 oxygen basis } \\
\hline $\mathrm{Si}$ & $2.15-3.16$ & 3.01 & 0.23 \\
\hline $\mathrm{Al}^{\mathrm{IV}}$ & $0.84-1.01$ & 0.93 & 0.06 \\
\hline$\Sigma Z=4$ & 4 & 4.00 & 0.00 \\
\hline${ }^{\mathrm{VI}} \mathrm{Al}$ & $1.48-1.65$ & 1.59 & 0.04 \\
\hline${ }^{\mathrm{VI}} \mathrm{Ti}$ & $0.00-0.04$ & 0.02 & 0.01 \\
\hline${ }^{\mathrm{VI}} \mathrm{Fe}^{2+}$ & $0.06-0.10$ & 0.08 & 0.01 \\
\hline${ }^{\mathrm{VI}} \mathrm{Mn}$ & $0.00-0.00$ & 0.00 & 0.00 \\
\hline${ }^{\mathrm{VI}} \mathrm{Mg}$ & $0.05-0.15$ & 0.09 & 0.03 \\
\hline $\mathrm{Li}$ & $1.12-1.35$ & 1.20 & 0.06 \\
\hline$\Sigma Y \sim 3$ & $2.90-3.10$ & 2.99 & 0.05 \\
\hline $\mathrm{Ca}$ & $0.00-0.00$ & 0.00 & 0.00 \\
\hline $\mathrm{Na}$ & $0.00-0.05$ & 0.03 & 0.02 \\
\hline $\mathrm{K}$ & $0.38-0.71$ & 0.47 & 0.08 \\
\hline$\Sigma \mathrm{X} \sim 1$ & $0.40-0.71$ & 0.50 & 0.08 \\
\hline $\mathrm{Fe}^{\#}$ & $0.41-0.69$ & 0.50 & 0.08 \\
\hline $\mathrm{Mg}^{\#}$ & $0.31-0.59$ & 0.50 & 0.08 \\
\hline
\end{tabular}


Table 5. (Continued.)

\begin{tabular}{|c|c|c|c|}
\hline & Range & Mean & Std. dev. \\
\hline \multicolumn{4}{|c|}{ (c) 13 samples Ms (TLg) } \\
\hline $\mathrm{SiO}_{2}$ & $45.86-50.71$ & 49.19 & 1.26 \\
\hline $\mathrm{TiO}_{2}$ & $0.11-0.35$ & 0.19 & 0.06 \\
\hline $\mathrm{Al}_{2} \mathrm{O}_{3}$ & $34.82-37.34$ & 36.60 & 0.63 \\
\hline $\mathrm{FeO}$ & $1.90-2.15$ & 2.02 & 0.08 \\
\hline $\mathrm{MnO}$ & $0.00-0.03$ & 0.01 & 0.01 \\
\hline $\mathrm{MgO}$ & $0.46-0.87$ & 0.55 & 0.11 \\
\hline $\mathrm{CaO}$ & $0.00-0.12$ & 0.03 & 0.03 \\
\hline $\mathrm{Na}_{2} \mathrm{O}$ & $0.04-0.42$ & 0.29 & 0.11 \\
\hline $\mathrm{K}_{2} \mathrm{O}$ & $3.12-6.97$ & 5.75 & 0.87 \\
\hline $\mathrm{Li}_{2} \mathrm{O}(\mathrm{c})$ & $3.61-5.00$ & 4.57 & 0.36 \\
\hline \multicolumn{4}{|c|}{ Structural formula based on 22 oxygen basis } \\
\hline $\mathrm{Si}$ & $3.00-3.05$ & 3.02 & 0.02 \\
\hline $\mathrm{Al}^{\mathrm{IV}}$ & $0.95-1.00$ & 0.98 & 0.02 \\
\hline$\Sigma Z=4$ & 4 & 4.00 & 0.00 \\
\hline${ }^{\mathrm{VI}} \mathrm{Al}$ & $1.65-1.70$ & 1.67 & 0.02 \\
\hline${ }^{\mathrm{VI}} \mathrm{Ti}$ & $0.01-0.02$ & 0.01 & 0.00 \\
\hline${ }^{\mathrm{VI}} \mathrm{Fe}^{2+}$ & $0.10-0.11$ & 0.11 & 0.01 \\
\hline${ }^{\mathrm{VI}} \mathrm{Mn}$ & $0.00-0.00$ & 0.00 & 0.00 \\
\hline${ }^{\mathrm{VI}} \mathrm{Mg}$ & $0.04-0.08$ & 0.05 & 0.01 \\
\hline $\mathrm{Li}$ & $0.95-1.21$ & 1.13 & 0.07 \\
\hline$\Sigma \mathrm{Y} \sim 3$ & $2.82-3.09$ & 2.97 & 0.06 \\
\hline $\mathrm{Ca}$ & $0.00-0.01$ & 0.00 & 0.00 \\
\hline $\mathrm{Na}$ & $0.00-0.05$ & 0.04 & 0.01 \\
\hline $\mathrm{K}$ & $0.24-0.55$ & 0.45 & 0.07 \\
\hline$\Sigma \mathrm{X} \sim 1$ & $0.24-0.60$ & 0.49 & 0.09 \\
\hline $\mathrm{Fe}^{\#}$ & $0.58-0.71$ & 0.68 & 0.03 \\
\hline $\mathrm{Mg}^{\#}$ & $0.29-0.42$ & 0.32 & 0.03 \\
\hline
\end{tabular}

$\mathrm{Fe}^{\#}=\mathrm{Fe}^{\mathrm{t}} / \mathrm{Fe}^{\mathrm{t}}+\mathrm{Mg} ; \mathrm{Mg}^{\#}=\mathrm{Mg} / \mathrm{Mg}+\mathrm{Fe}^{\mathrm{t}}$.

Note: The entire database can be obtained from the corresponding author. respectively. Plagioclase from $\operatorname{TLg}\left(\mathrm{An}_{2}-\mathrm{An}_{15}\right)$ is mostly oligoclase, although a few are albite (figure 4); however, rim composition is slightly more anorthitic compared to the core suggesting reverse zoning. $\mathrm{K}$-feldspar in $\mathrm{TLg}\left(\mathrm{Or}_{87}\right.$ to $\left.\mathrm{Or}_{94}\right)$ is mostly sanidine to orthoclase. Representative analyses of biotite from TLg are given in table 4(c). The biotite from $\mathrm{TLg}\left(\mathrm{Mg} / \mathrm{Mg}+\mathrm{Fe}^{\mathrm{t}}=0.25-0.30\right)$ are classified as ferrobiotites enriched in siderophyllite component and buffered mostly between FMQ and NNO (figure 5). TLg biotites show compositional affinity with biotite syncrystallised with muscovite (De Albuquerque 1973). Dominant $3 \mathrm{Fe} \rightleftharpoons 2 \mathrm{Al}$ and $3 \mathrm{Mg} \rightleftharpoons 2 \mathrm{Al}$ substitutions (figure $6 \mathrm{a}$, b) and large variations in the $\mathrm{Fe}^{\mathrm{t}} / \mathrm{Fe}^{\mathrm{t}}+\mathrm{Mg}$ ratios $(\mathrm{TLg}=0.70-0.75)$ in the biotite from TLg suggests its peraluminous (S-type) and reduced nature. Representative analyses of muscovites from TLg and their empirically estimated Li-contents (Tindle and Webb 1990; Tischendorf 1997; Tischendorf et al. 1999; Yavuz 2001) are given in table 5(c). Muscovite in TLg $\left(\mathrm{Mg} / \mathrm{Mg}+\mathrm{Fe}^{\mathrm{t}}=0.29\right.$ 0.53) represent celadonitic and paragonitic solidsolutions (Miller et al. 1981), belonging to the Li-Al mica group (lithian muscovite, lepidolite and zinnwaldite, figure 7). Representative analyses of tourmaline from TLg are given in table $6(\mathrm{~b}) . \mathrm{Fe}^{3+}$ and $\mathrm{Fe}^{2+}$ were obtained from total iron by partition using the tourmal program (Yavuz 1997). It has been assumed that boron has a stoichiometric abundance of 3 apfu (e.g., Torres-Ruiz et al. 2003). Tourmaline from TLg belong principally to the vacancy and alkali groups (Hawthorne and Henry 1999) containing minor amounts of Mn (tsiliasite component; Slivko 1959), but lack absolutely any empirically estimated $\mathrm{Fe}^{3+}$ content, suggesting

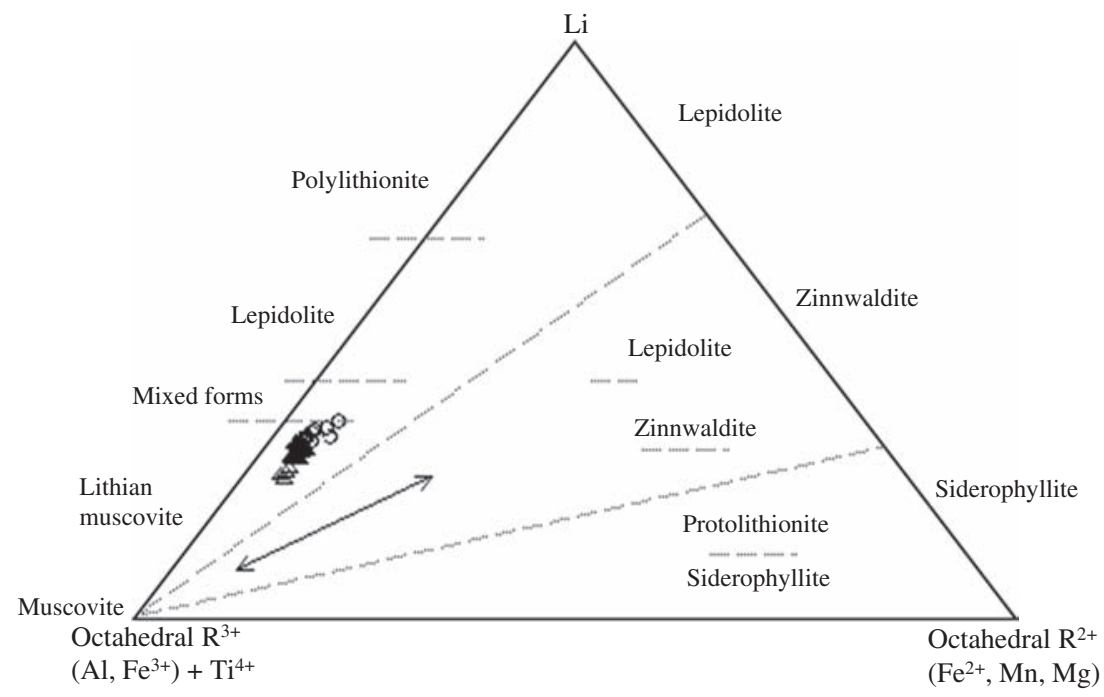

Figure 7. Classification of muscovite from GGn $(+)$, EPG $(\bigcirc)$ and TLg $(\Delta)$ on a ternary $\mathrm{R}^{3+}-\mathrm{Li}-\mathrm{R}^{2+}$ diagram $($ Foster 1960; Monier and Robert 1986; Stone et al. 1986). The solid line with arrow heads represents a transitional series between muscovite and zinnwaldite (Monier and Robert 1986). 
Table 6. Representative electron microprobe analysis of tourmaline from (a) Early Palaeozoic granitoids (EPG) and, (b) tourmaline bearing leucogranite (TLg).

\begin{tabular}{lcrr}
\hline \multicolumn{1}{c}{ Range } & Mean & Std. de \\
\hline (a) 6 samples Tur (EPG) & & \\
$\mathrm{SiO}_{2}$ & $31.48-39.48$ & 34.48 & 2.72 \\
$\mathrm{Al}_{2} \mathrm{O}_{3}$ & $19.33-29.30$ & 24.97 & 4.04 \\
$\mathrm{TiO}_{2}$ & $0.08-0.54$ & 0.31 & 0.17 \\
$\mathrm{FeO}$ & $8.44-11.78$ & 10.51 & 1.24 \\
$\mathrm{MnO}$ & $0.07-0.14$ & 0.11 & 0.02 \\
$\mathrm{MgO}$ & $1.69-2.38$ & 2.05 & 0.27 \\
$\mathrm{CaO}$ & $0.11-0.32$ & 0.22 & 0.09 \\
$\mathrm{Na}_{2} \mathrm{O}$ & $0.58-1.76$ & 1.21 & 0.52
\end{tabular}

Structural formula based on 24.5 oxygen basis

\begin{tabular}{|c|c|c|c|}
\hline $\mathrm{Si}$ & 6.00 & 6.00 & 0.00 \\
\hline $\mathrm{Al}^{\mathrm{IV}}$ & 0.00 & 0.00 & 0.00 \\
\hline$\Sigma \mathrm{T}$ & 6.00 & 6.00 & 0.00 \\
\hline B & 3.00 & 3.00 & 0.00 \\
\hline $\mathrm{Al}(\mathrm{Z})$ & $5.65-6.00$ & 5.91 & 0.15 \\
\hline $\mathrm{Mg}$ & $0.00-0.35$ & 0.10 & 0.15 \\
\hline$\Sigma Z$ & 6.00 & 6.00 & 0.00 \\
\hline $\mathrm{Al}(\mathrm{Y})$ & $0.00-0.26$ & 0.07 & 0.10 \\
\hline $\mathrm{Ti}$ & $0.01-0.09$ & 0.05 & 0.03 \\
\hline $\mathrm{Fe}^{2+}$ & $0.61-1.76$ & 1.30 & 0.40 \\
\hline $\mathrm{Mg}$ & $0.26-0.64$ & 0.50 & 0.13 \\
\hline $\mathrm{Mn}$ & $0.01-0.02$ & 0.02 & 0.00 \\
\hline$\Sigma Y$ & $1.22-2.60$ & 1.92 & 0.49 \\
\hline $\mathrm{Ca}$ & $0.02-0.07$ & 0.05 & 0.02 \\
\hline $\mathrm{Na}$ & $0.21-0.70$ & 0.46 & 0.21 \\
\hline$\square$ & $0.24-0.77$ & 0.49 & 0.23 \\
\hline$\Sigma \mathrm{X}$ & 1.00 & 1.00 & 0.00 \\
\hline $\mathrm{Fe} / \mathrm{Fe}+\mathrm{Mg}$ & $0.71-0.77$ & 0.74 & 0.02 \\
\hline $\mathrm{Na} / \mathrm{Na}+\mathrm{Ca}$ & $0.87-0.93$ & 0.91 & 0.02 \\
\hline \multicolumn{4}{|c|}{ (b) 7 samples Tur (TLg) } \\
\hline $\mathrm{SiO}_{2}$ & $32.79-36.60$ & 34.73 & 1.62 \\
\hline $\mathrm{Al}_{2} \mathrm{O}_{3}$ & $24.32-34.83$ & 29.37 & 4.11 \\
\hline $\mathrm{TiO}_{2}$ & $0.17-0.45$ & 0.30 & 0.12 \\
\hline $\mathrm{FeO}$ & $10.67-12.22$ & 11.51 & 0.52 \\
\hline $\mathrm{MnO}$ & $0.09-0.19$ & 0.15 & 0.04 \\
\hline $\mathrm{MgO}$ & $1.46-3.11$ & 2.43 & 0.56 \\
\hline $\mathrm{CaO}$ & $0.12-0.32$ & 0.21 & 0.08 \\
\hline $\mathrm{Na}_{2} \mathrm{O}$ & $0.85-1.88$ & 1.12 & 0.42 \\
\hline
\end{tabular}

Structural formula based on 24.5 oxygen basis

\begin{tabular}{|c|c|c|c|}
\hline $\mathrm{Si}$ & $5.91-6.00$ & 5.99 & 0.03 \\
\hline $\mathrm{Al}^{\mathrm{IV}}$ & $0.00-0.09$ & 0.01 & 0.03 \\
\hline$\Sigma \mathrm{T}$ & 6.00 & 6.00 & 0.00 \\
\hline B & 3.00 & 3.00 & 0.00 \\
\hline $\mathrm{Al}(\mathrm{Z})$ & $5.72-6.00$ & 5.96 & 0.11 \\
\hline $\mathrm{Mg}$ & $0.00-0.26$ & 0.04 & 0.10 \\
\hline$\Sigma \mathrm{Z}$ & 6.00 & 6.00 & 0.00 \\
\hline $\mathrm{Al}(\mathrm{Y})$ & $0.00-0.72$ & 0.29 & 0.30 \\
\hline $\mathrm{Ti}$ & $0.02-0.06$ & 0.04 & 0.02 \\
\hline $\mathrm{Fe}^{2+}$ & $1.22-1.89$ & 1.62 & 0.22 \\
\hline $\mathrm{Mg}$ & $0.41-0.85$ & 0.62 & 0.14 \\
\hline $\mathrm{Mn}$ & $0.01-0.03$ & 0.02 & 0.01 \\
\hline$\Sigma Y$ & $2.00-2.96$ & 2.59 & 0.36 \\
\hline
\end{tabular}

Table 6. (Continued.)

\begin{tabular}{llcc}
\hline & Range & Mean & Std. dev. \\
\hline $\mathrm{Ca}$ & $0.02-0.07$ & 0.04 & 0.02 \\
$\mathrm{Na}$ & $0.29-0.60$ & 0.38 & 0.12 \\
$\square$ & $0.38-0.67$ & 0.58 & 0.11 \\
$\Sigma \mathrm{X}$ & 1.00 & 1.00 & 0.00 \\
$\mathrm{Fe} / \mathrm{Fe}+\mathrm{Mg}$ & $0.68-0.82$ & 0.73 & 0.05 \\
$\mathrm{Na} / \mathrm{Na}+\mathrm{Ca}$ & $0.83-0.97$ & 0.89 & 0.05 \\
\hline
\end{tabular}

Note: The entire database can be obtained from the corresponding author.

its affinity with schorl-elbaite group (figure 8). In terms of $\mathrm{Al}-\mathrm{Fe}^{\mathrm{t}}-\mathrm{Mg}$ (Henry and Guidotti 1985) the tourmaline from TLg appear to have crystallised from a lithium-poor, tur-bt granitic melt or its derivative pegmatites and aplites (figure 9).

\section{Whole rock and trace element geochemistry}

The mineral assemblage (ms-bt-pl-kf-qtz) and geochemical features of GGn (mol. $\mathrm{A} / \mathrm{CNK}=$ 1.00-1.75), EPG (mol. A/CNK $=1.00-1.25)$ and TLg (mol. A/CNK $=1.08-2.80$ ) suggest typical peraluminous (S-type) granites (figure 10) as defined by Chappell and White (1974) and Debon and Le Fort (1983). Major oxide content of seven GGn samples are given in table 7(a). The GGn is characterised by moderate to high $\mathrm{K}_{2} \mathrm{O}$ (1.90-4.90 $\mathrm{wt} \%), \mathrm{Al}_{2} \mathrm{O}_{3}$ (14.11-15.79 wt\%) and $\mathrm{MgO}(0.52-$ $1.28 \mathrm{wt} \%)$ contents over a narrow range of $\mathrm{SiO}_{2}$ (68.15-73.00 wt\%). $\mathrm{CaO}, \mathrm{TiO}_{2}$ and $\mathrm{Fe}_{2} \mathrm{O}_{3}^{\mathrm{t}}$ show a broadly negative correlation with $\mathrm{SiO}_{2}$ for most GGn samples, but $\mathrm{Al}_{2} \mathrm{O}_{3}, \mathrm{Na}_{2} \mathrm{O}$ and $\mathrm{K}_{2} \mathrm{O}$ show slight scatter (figure $11 \mathrm{a}-\mathrm{f}$ ). The major oxide contents of fifteen EPG samples are given in table 7(b). The EPG shows high $\mathrm{Al}_{2} \mathrm{O}_{3}$ (13.09-14.82 wt\%) and low to high $\mathrm{MgO}(0.24-1.33 \mathrm{wt} \%)$ over a narrow $\mathrm{SiO}_{2}$ range (69.48-74.99 wt\%). $\mathrm{TiO}_{2}, \mathrm{Al}_{2} \mathrm{O}_{3}$, $\mathrm{Fe}_{2} \mathrm{O}_{3}^{\mathrm{t}}, \mathrm{CaO}$, and, to a certain extent, $\mathrm{K}_{2} \mathrm{O}$ show a broadly decreasing linear concentration with increasing $\mathrm{SiO}_{2}$ content (figure 11a-d, f), this may be accounted for by the early crystallisation of feldspars, mica (muscovite and biotite), apatite and iron oxides. $\mathrm{Na}_{2} \mathrm{O}$ does not exhibit any definite correlation with $\mathrm{SiO}_{2}$ (figure 11e). Scattering of some data points probably reflects heterogeneous modal mineral abundance. Major oxides of eight TLg samples are given in table $7(\mathrm{a})$, which show a relatively wider range of $\mathrm{SiO}_{2}$ (71.4377.37 wt\%) as compared to GGn and EPG. CaO, $\mathrm{K}_{2} \mathrm{O}, \mathrm{Fe}_{2} \mathrm{O}_{3}^{\mathrm{t}}$, and $\mathrm{TiO}_{2}$ show negative curvilinear trends against $\mathrm{SiO}_{2}$ whereas $\mathrm{Na}_{2} \mathrm{O}$ exhibits strong positive correlation with $\mathrm{SiO}_{2}$ (figure 11a-f). $\mathrm{Al}_{2} \mathrm{O}_{3}$ shows scatter of data points. Trace elements of seven GGn samples are given in table 8(a).Th 


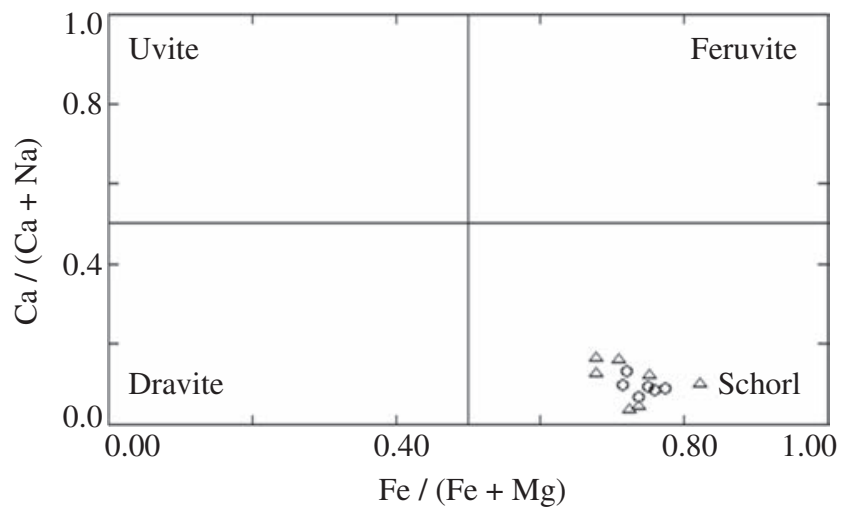

Figure 8. EPG $(\bigcirc)$ and TLg $(\Delta)$ tourmalines in terms of $\mathrm{Ca} /(\mathrm{Ca}+\mathrm{Na})$ vs. $\mathrm{Fe} /(\mathrm{Fe}+\mathrm{Mg})$ showing affinity with schorl group (London and Manning 1995).

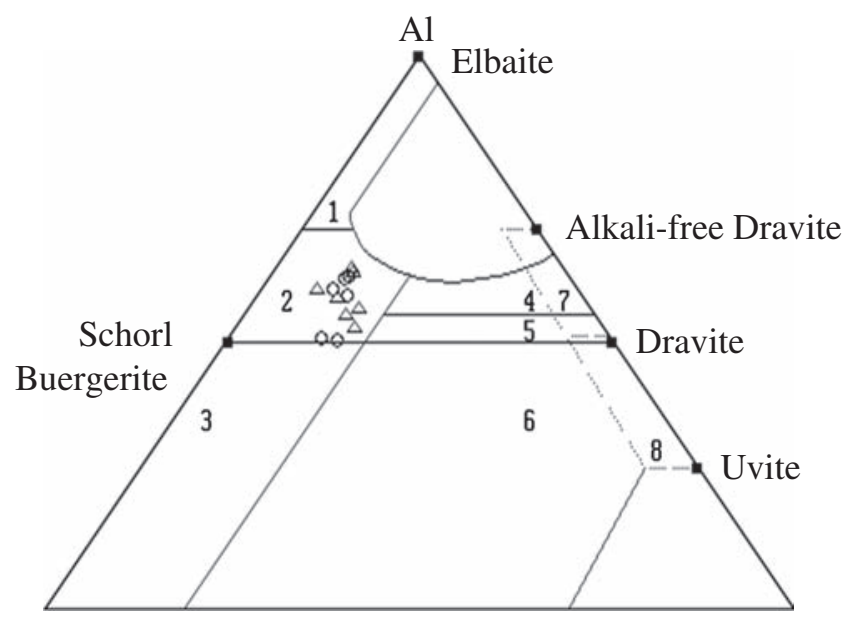

Fe (tot)

$\mathrm{Mg}$

Figure 9. $\mathrm{Fe}^{\mathrm{t}}-\mathrm{Al}-\mathrm{Mg}$ ternary diagram showing affinity of tourmaline from the EPG $(\bigcirc)$ and TLg $(\Delta)$ with lithiumpoor granitoids and associated felsic derivatives (field 2). Other fields are 1: Li-poor granitoid pegmatites and aplites, 2: Lithium-poor granitoids and associated felsic derivatives. $3: \mathrm{Fe}^{3+}$ rich quartz-tourmaline rocks (hydrothermally altered granites), 4: Metapelites and metapsammites coexisting with an Al-saturating phase, 5: Metapelites and metapsammites not coexisting with an Al-saturating phase, 6: $\mathrm{Fe}^{3+}$ rich quartz-tourmaline rocks, calc-silicate rocks and metapelites, 7: Low-Ca metaultramafics and Cr, V-rich metasediments, and 8: Metacarbonates and metapyroxenites (after Henry and Guidotti 1985).

shows negative correlation with $\mathrm{SiO}_{2}$ (figure 11j), whereas curvilinear antipathic correlation can be observed for $\mathrm{Rb} / \mathrm{Sr}$ vs. Sr (figure 110). Sr shows slight positive correlation with $\mathrm{SiO}_{2}$ (figure 11i). Wide data scatter can be observed for $\mathrm{Ba}, \mathrm{Nb}, \mathrm{Y}$, $\mathrm{Zr}$ versus $\mathrm{SiO}_{2}$ (figure $11 \mathrm{~h}, \mathrm{k}, \mathrm{l}, \mathrm{m}$ ) whereas $\mathrm{Rb}$ clusters in an area (figure $11 \mathrm{~g}$ ). Primitive mantle normalised multi elemental variation diagram for GGn (figure 12b) show lesser degree of variations for most elements compared to that of EPG and TLg, except Ta which varies widely in GGn and TLg (figure 12b, c). REE patterns for GGn (figure 13b) show uniform LREE and MREE

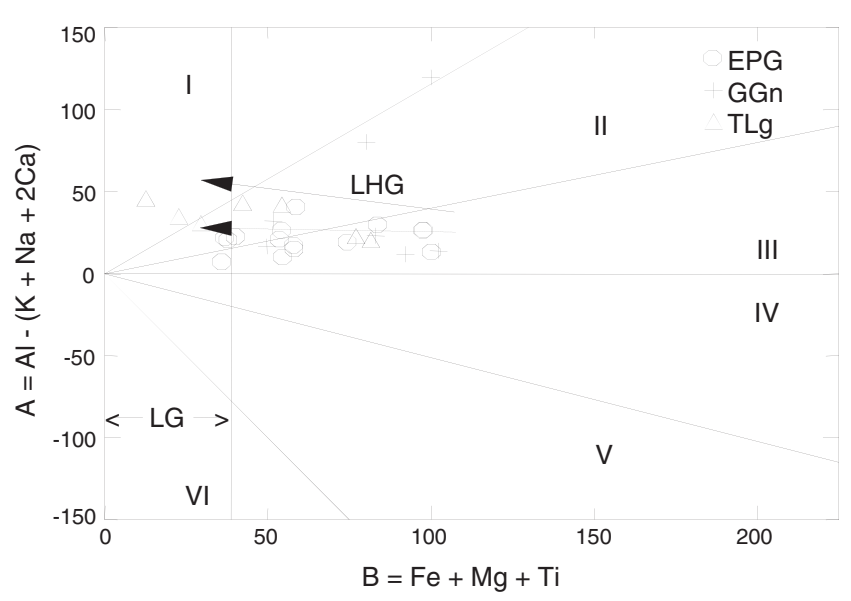

Figure 10. (a-b) Multicationic diagram (Debon and Le Fort 1983) plotted for EPG, GGn and TLg. The sectors I, II and III represent peraluminous, where $\mathrm{I}=$ muscovite $>$ biotite (by volume), II=biotite $>$ muscovite, III=biotite (usually alone, in some cases with minor amphibole). Fields IV, V and VI are for metaluminous rocks, where IV=biotite+amphibol \pm pyroxene, $V=$ clinopyroxene \pm amphibole \pm biotite, $\mathrm{VI}=$ unusual rocks (e.g., carbonatites). LG denotes the leucogranite field. Arrow with solid line shows the trend of Lesser Himalayan granitoids from Nepal-India-Pakistan (Le Fort et al. 1986a in Barbarin 1996). Arrow with dotted line shows EPG evolutionary trend.

(La to Ho) followed by flat HREE (Ho to Lu) patterns $\left(\mathrm{La}_{\mathrm{N}} / \mathrm{Lu}_{\mathrm{N}}=13.9\right.$ to 59.6) with moderate negative $\mathrm{Eu}$ anomalies $\left(\mathrm{Eu}_{\mathrm{N}} / \mathrm{Eu}^{*}=0.52\right.$ to 0.61$)$. Trace and REE contents (ppm) of 15 EPG samples are given in table $8(\mathrm{~b})$. Large-ion lithophile elements (LILE) such as $\mathrm{Ba}, \mathrm{Rb}, \mathrm{Sr}$ and high-field strength elements (HFSE) such as Zr, Y, Th, Nb, $\mathrm{U}$, and Ta show wide variations in their contents. $\mathrm{Sr}, \mathrm{Nb}, \mathrm{Zr}$ and $\mathrm{Ga}$ have broadly negative correlation with increasing $\mathrm{SiO}_{2}$ (figure 11i, k, m, n). Data are scattered for $\mathrm{Rb}, \mathrm{Ba}$, Th, and $\mathrm{Y}$ against $\mathrm{SiO}_{2}$ and show no correlation (figure $11 \mathrm{~g}, \mathrm{~h}, \mathrm{j}, \mathrm{l}$ ). In particular, $\mathrm{Rb}$ and $\mathrm{Ba}$ show a large range of variation for a given silica content, probably because of modal variations in biotite and K-feldspar, respectively, from sample to sample. Curvilinear antipathic correlation between $\mathrm{Rb} / \mathrm{Sr}$ and $\mathrm{Sr}$ (figure 11o) is observed. Mantle-normalised spidergrams show elevated content (10-100 times) for most elements relative to the mantle (figure 12a). Both $\mathrm{P}$ and Ti exhibit strong negative anomalies, whereas $\mathrm{Ba}, \mathrm{U}$ and $\mathrm{Th}$ show wide range of variations. Chondrite-normalised LREE and MREE seem coherent (Pr-Ho) then HREE (Ho-La) flatten out, but most notably the LREE (La and $\mathrm{Ce}$ ) seem to be depleted (figure 13a). Moderate degree of LREE to HREE fractionation $\left(\mathrm{La}_{\mathrm{N}} / \mathrm{Lu}\right.$ 5.6-35.3) and negative $\mathrm{Eu}$ anomalies $\left(\mathrm{Eu}_{\mathrm{N}} / \mathrm{Eu}^{*}=\right.$ $0.35-0.64)$ can be noticed. Trace elements of eight TLg samples are given in table 8(a). Relatively wider variations of $\mathrm{Rb} / \mathrm{Sr}$ and $\mathrm{Sr} / \mathrm{Ba}$ ratios of TLg can be observed as compared to 


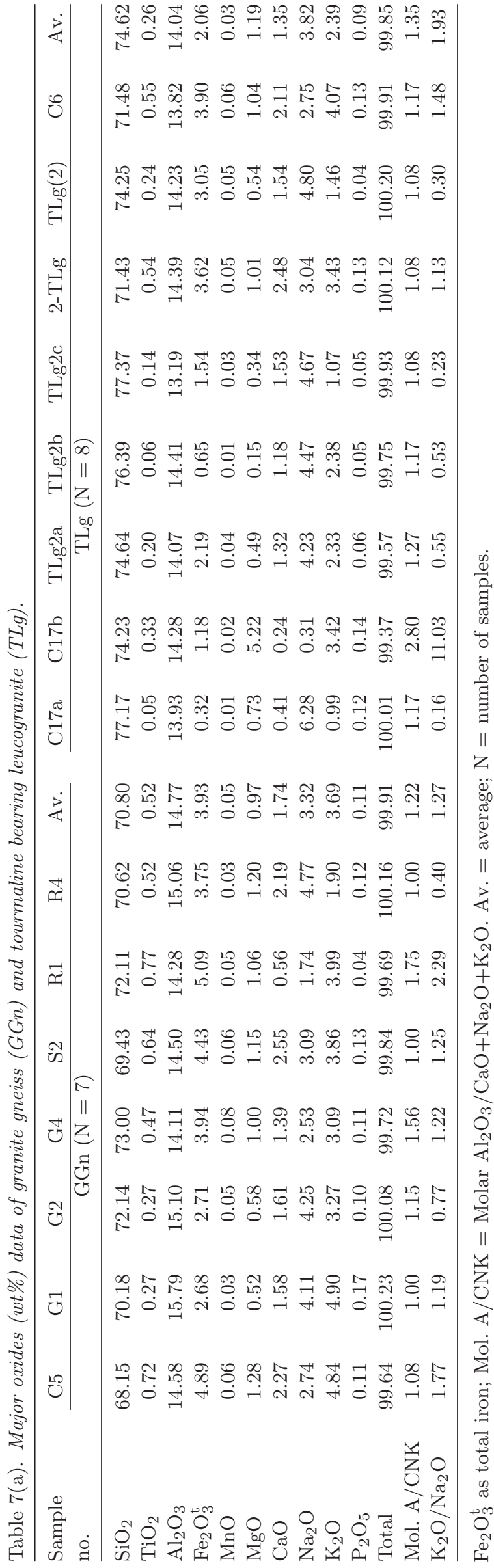

\begin{tabular}{|c|c|}
\hline 安空 & 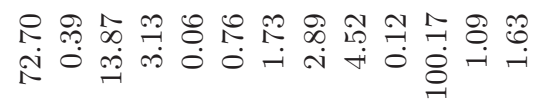 \\
\hline $\mathscr{\imath}$ & 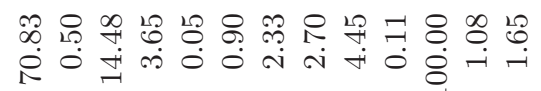 \\
\hline జै & 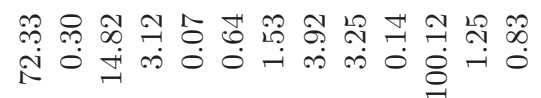 \\
\hline $\bar{\sigma}$ & 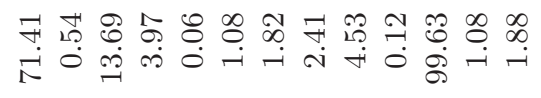 \\
\hline 今ิ & 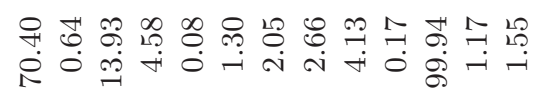 \\
\hline ֶ̃ & 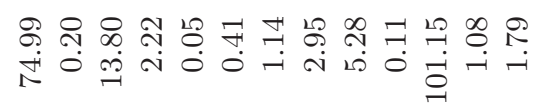 \\
\hline$\stackrel{\overbrace{}}{\partial}$ & 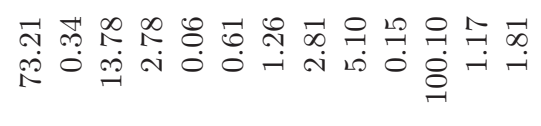 \\
\hline$\stackrel{\circ}{\circlearrowright}$ & 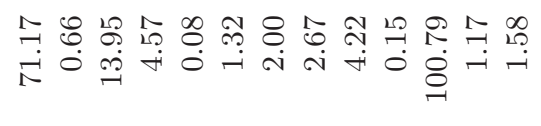 \\
\hline 8 & 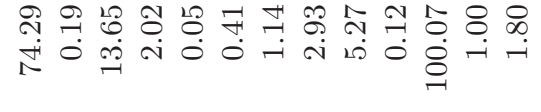 \\
\hline 己 & 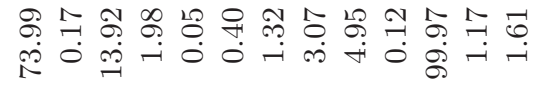 \\
\hline $\bar{U}$ & 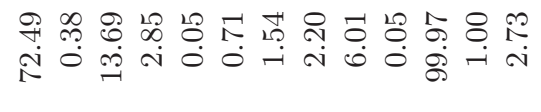 \\
\hline$\stackrel{0}{4}$ & 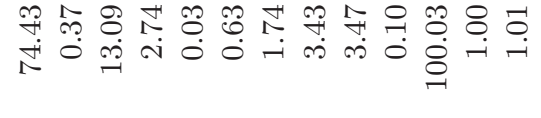 \\
\hline $\mathbb{Z}$ & 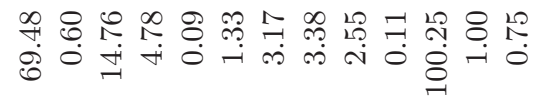 \\
\hline$\stackrel{m}{4}$ & 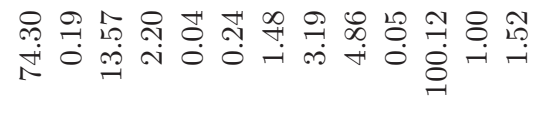 \\
\hline$\frac{\tau}{4}$ & 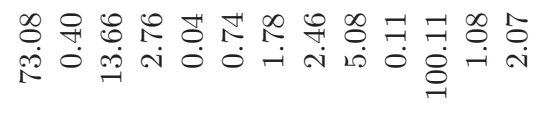 \\
\hline & 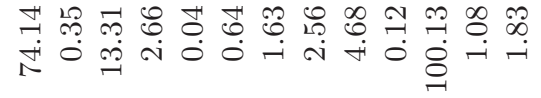 \\
\hline & 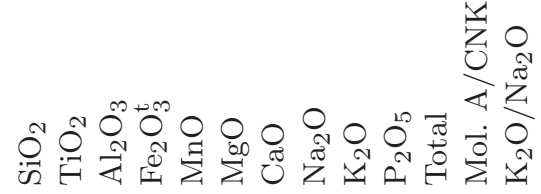 \\
\hline
\end{tabular}


EPG. However, variation diagrams for TLg show some similarities with EPG. Rb, Ba, Nb, Y and $\mathrm{Zr}$ show negative correlation with $\mathrm{SiO}_{2}$ (figure $11 \mathrm{~g}, \mathrm{~h}, \mathrm{k}, \mathrm{l}, \mathrm{m})$. A wide scatter can be observed for $\mathrm{Sr}$ and $\mathrm{Th}$ contents against $\mathrm{SiO}_{2}$ (figure 11i, j). The mantle-normalised spidergrams of TLg show much more elemental variation when compared to EPG and GGn and, therefore could be considered characteristic of Himalayan leucogranites (figure 12c). The observed trace elements characteristic of TLg might be a reflection of progressively evolving nature of crustal protoliths in space and time and its recycling during geological episodes, referred elsewhere as tertiary peraluminous granitoids (Clarke 1992). One garnetbearing TLg (A5) bears elevated U and depleted $\mathrm{Ba}$ and $\mathrm{Nb}$ contents as compared to other TLg. Slightly inclined to flat LREE patterns and moderate to gently sloping HREE patterns with moderate negative $\mathrm{Eu}$ anomalies $\left(\mathrm{Eu}_{\mathrm{N}} / \mathrm{Eu}^{*}=0.32-0.46\right)$ can be observed (figure 13c). La and Ce seem depleted in TLg and the HREE decreases rather more abruptly than in EPG. LREE to HREE fractionations $\left(\mathrm{La}_{\mathrm{N}} / \mathrm{Lu}_{\mathrm{N}}=3.2-32.1\right)$ of TLg are larger as compared to EPG and GGn. Enrichment of HREE is obvious in one garnet-bearing TLg (C6).

\subsection{Tectonic setting}

Syn-collisional tectonic affinity for GGn, EPG and TLg can be very well constrained on compositiontectonic plot (figure 14). In terms of trace elements (Rb, Nb, Y), GGn and EPG cluster at the boundary between VAG and syn-COLG granites, whereas the TLg spread over VAG to the syn-COLG fields (figure 15). However, on the normalised patterns of ocean ridge granite (ORG) (not shown) GGn, EPG and TLg all show high contents of Rb, Th and low Ce and Hf, which are characteristics of syncollisional granites (Pearce et al. 1984). Dominant but to various degrees of crustal components in the genesis of all three granitoids can be inferred by the low contents of $\mathrm{Ta}$ and $\mathrm{Nb}$ relative to $\mathrm{Rb}$ in each.

\section{Petrogenetic discussion}

\subsection{Field petrography, nature of felsic melts, granite series and evolution of mineral phases}

The development of some local migmatitic components close to leucocratic and melanocratic layers in GGn and EPG appears to be synchronous with the Himalayan orogeny-induced compressional forces, which have formed melanosome and leucosome (haplogranite) components (Mehnert 1968). The presence of melanosome adjacent to each leucocratic layer is a common feature, and suggests in situ formation rather than the migration of melt (Collins and Sawyer 1996). The presence of leucocratic and melanocratic bands near to a shear zone may however suggest synplutonic segregation of melt from crystallising pluton rather than partial melting (E W Sawyer, pers. comm.). The quartzofeldspathic megacrysts in EPG represent phenocrysts and not porphyroblasts because the K-feldspar megacrysts exhibit growth, twinning, shape, inclusion and dissolution features that are characteristics of magmatic processes (e.g., Vernon 1986). The presence of two micas (Ms-Bt) in GGn, EPG and TLg suggest that the granitic melts from which they crystallised were water rich, or even water-saturated melt (sensu stricto) and generated within a narrow temperature interval, by the partial melting of fusible crustal components (Wyllie 1997). Therefore they cluster within the anatectic granite field (Laymere and Bowden 1982) close to the granite eutectic (Harris and Inger 1992).

The range of variation in the magnetic susceptibility (GGn: $0.052-0.220 \times 10^{-3}$ SI; EPG: $0.016-0.187 \times 10^{-3}$ SI; TLg: $0.011-0.088 \times 10^{-3}$ SI) is strongly suggestive of the ilmenite series for these felsic magmas with a range of crustal residence times. Bulk MS values of GGn $(\chi=0.168-0.190$ $\left.\times 10^{-3} \mathrm{SI}\right)$ and EPG $\left(\chi=0.096-0.130 \times 10^{-3} \mathrm{SI}\right)$ indicate moderately reduced rocks, whereas TLg $\left(\chi=0.054-0.056 \times 10^{-3} \mathrm{SI}\right)$ appear to be strongly reduced, which are mostly intrinsic to the source lithology (e.g., Ishihara 1977). The felsic magmatism from each of the different episodes (Neoproterozoic, Early Palaeozoic to Himalayan), thus show a trend of increasing reduction and were probably generated in the middle of the continental crust from source rocks with low $\mathrm{Fe}_{2} \mathrm{O}_{3} / \mathrm{FeO}$ ratios (Ishihara 1984), and/or reduction was due to the carbon contained in the pelitic protoliths (Ishihara and Matsuhisa 1999).

Most plagioclases from GGn $\left(\mathrm{An}_{10}-\mathrm{An}_{31}\right)$, EPG $\left(\mathrm{An}_{15}-\mathrm{An}_{33}\right)$ and TLg $\left(\mathrm{An}_{2}-\mathrm{An}_{15}\right)$ have crystallised from felsic melt. The reverse compositional zoning of the plagioclase is a result of the changing water pressure on the liquidus (e.g., Yoder et al. 1957 in Vance 1965; Wiebe 1968), or the reduced undercooling, or a build-up of volatiles in the felsic melt (Loomis 1982). Most crystals of K-feldspar in GGn, EPG and TLg represent sanidine to orthoclase and are related to crystallisation of the felsic magmas, but some of them lack any anorthite component, possibly because they re-equilibrated at near solidus or subsolidus condition.

The sum of X-site occupancy more than unity in some biotites from GGn, EPG and TLg may be due to the replacement of $\mathrm{K}$ by $\mathrm{Ba}, \mathrm{Rb}$ and $\mathrm{Cs}$ (Deer et al. 1963). Substitutions such as $3 \mathrm{Mg} \rightleftharpoons 2 \mathrm{Al}$ and $3 \mathrm{Fe} \rightleftharpoons 2 \mathrm{Al}$ together with an average $\mathrm{FeO}^{\mathrm{t}} / \mathrm{MgO}$ 

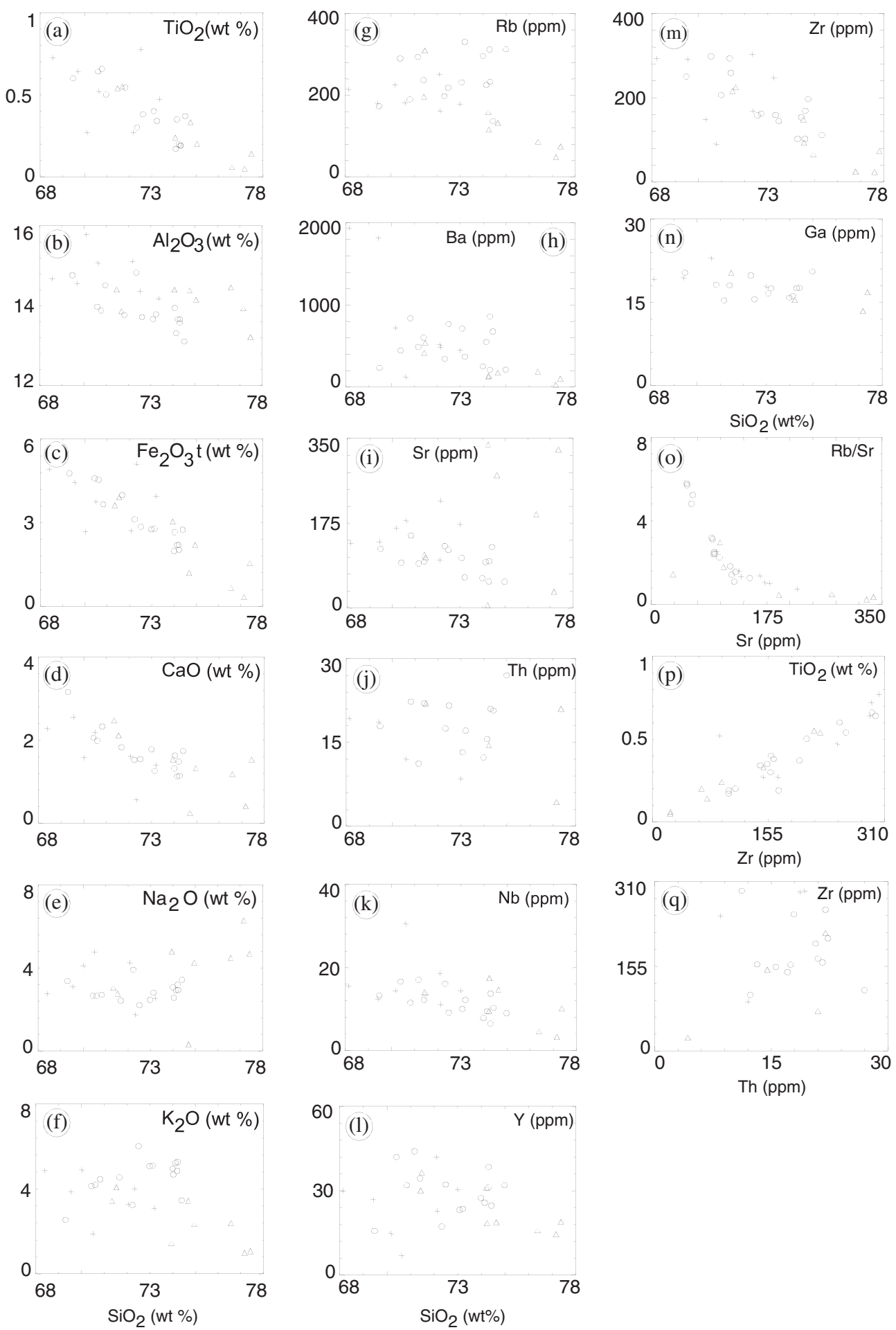

Figure 11. (a-q) Major oxide and trace element vs. $\mathrm{SiO}_{2}$ variation diagrams for GGn $(+)$, EPG $(\bigcirc)$ and TLg $(\Delta)$ granite suites.

$=2.42$ for $\mathrm{GGn}, \mathrm{FeO}^{\mathrm{t}} / \mathrm{MgO}=3.54$ for $\mathrm{EPG}$, and $\mathrm{FeO}^{\mathrm{t}} / \mathrm{MgO}=4.73$ in the TLg indicate that the biotites are characteristic of crystallisation from a peraluminous (S-type) felsic magma suite (AbdelRahman 1994), and are similar to those observed from the Strathbogie (S-type granite) batholith of Australia (Burkhard 1991). The $3 \mathrm{Fe} \rightleftharpoons 2 \mathrm{Al}$ substitution predominates over $3 \mathrm{Mg} \rightleftharpoons 2 \mathrm{Al}$ in $\mathrm{GGn}, \mathrm{EPG}$ and TLg, suggesting low lithium contents (estimated) in the biotites, and can therefore be termed as ferrous lithium mica because of the minor $\mathrm{Li} \rightleftharpoons \mathrm{Fe}$ and $\mathrm{Li} \rightleftharpoons \mathrm{Ti}$ substitutions.

The trend to increased reduction in the felsic melts, inferred from $100 * \mathrm{Fe} / \mathrm{Fe}+\mathrm{Mg}$ ratios (Wones and Eugster 1965) of biotite (GGn = $55-66, \mathrm{EPG}=53-73, \mathrm{TLg}=70-75)$ can be primarily 
Table 8(a). Trace element (ppm) and elemental ratios of granite gneiss (GGn,7 samples) and tourmaline bearing leucogranite (TLg, 8 samples).

\begin{tabular}{|c|c|c|c|c|c|c|c|c|c|c|c|c|c|c|c|}
\hline Samp & C5 & G4 & $\mathrm{S} 2$ & $\mathrm{R} 4$ & G1 & G2 & $\mathrm{R} 1$ & $\mathrm{C} 17 \mathrm{a}$ & $\mathrm{C} 17 \mathrm{~b}$ & TLg-2c & $\mathrm{C} 6$ & ГLg-2a & $L g-2 b$ & TLg2 & 2-TLg \\
\hline & & & & GGn & & & & & & & & & & & \\
\hline $\mathrm{Ga}$ & 19.1 & 17.7 & 19.4 & 22.9 & 13.6 & 13.7 & 18.3 & 13.4 & 15.4 & 16.8 & 20.3 & 13.4 & 12.6 & 14.7 & 13 \\
\hline $\mathrm{b}$ & 214.1 & 178.6 & 180.4 & 181 & 225.1 & 161.6 & 250.9 & 8.3 & 158.4 & 74.3 & 309.2 & 131.8 & 85.9 & 115.7 & 196.3 \\
\hline & 133.8 & 172.5 & 136.8 & 179.8 & 165.2 & 221.4 & 99.1 & 33.6 & 6 & 326.7 & 104.5 & & 193.6 & 337 & 109.8 \\
\hline$Y$ & 29.9 & 30.4 & 26.8 & 6.9 & 14.8 & 22.8 & 42 & 14.5 & 31.1 & 18.9 & 36.5 & 18.8 & 15.9 & 18.4 & 30.1 \\
\hline $\mathrm{Zr}$ & 292.5 & 247.1 & 290.3 & 90 & 148.1 & 168.2 & 302.6 & 23.9 & 148.3 & 73.1 & 216 & 65.8 & 24.4 & 92.9 & 224.4 \\
\hline b & 15.5 & 14.4 & 12.4 & 30.5 & 14.4 & 11.1 & 18.6 & 3.3 & 9.5 & 10.1 & 14 & 14.6 & 4.6 & 17.4 & 14 \\
\hline 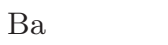 & 1939.6 & 447.2 & 1816.6 & 124.1 & 721.1 & 486 & 511.7 & 26.3 & 125.8 & 98.3 & 537 & 172.7 & 185 & 137.9 & 417.1 \\
\hline$a$ & 1.2 & 1.1 & 1.2 & 3 & 1.4 & 1.8 & 0.8 & 0.7 & 0.4 & 1.8 & 1.2 & 3.4 & 0.5 & 1 & 0.4 \\
\hline $\mathrm{h}$ & 19.3 & 8.5 & 18.7 & 12 & 9.2 & 10.1 & 12.8 & 4.3 & 14.5 & 21 & 22.2 & 11.8 & 4.3 & 9.2 & 9.4 \\
\hline & 2.6 & 2.5 & 2.6 & 4.4 & 3 & 3.6 & 2.1 & 1.2 & 1.7 & 2.2 & 1.6 & 2 & 1.1 & 2 & 1.6 \\
\hline & & 14 & & 87 & 180 & 168 & 132 & 170 & 179 & 119 & 161 & 146 & 229 & 104 & 145 \\
\hline & & 1. & 2 & 1.0 & & 0.73 & 2.53 & & .4 & & 96 & & & 0.34 & 1.79 \\
\hline $\mathrm{b} / \mathrm{Ba}$ & 0.11 & 0.4 & 0.1 & 1.46 & 0.31 & 0.33 & 0.49 & 1.84 & 1.26 & 0.76 & 0.58 & 0.76 & 16 & 0.84 & 0.47 \\
\hline $\mathrm{r} / \mathrm{Ba}$ & .07 & 0.39 & 0.08 & 1.45 & 0.23 & 0.46 & 0.19 & 1.28 & 0.05 & 3.32 & 0.19 & 1.59 & .05 & 2.44 & 0.26 \\
\hline $\mathrm{i} / \mathrm{P}$ & & 26 & 7 & 6.16 & 2.26 & 3.84 & 27.36 & & 3.35 & 1.33 & 6.01 & 4.74 & & 8.53 & 6.09 \\
\hline & & 26.85 & & 28.66 & 26.83 & 28.56 & 35.12 & & & & 30.87 & & & 28.69 & 25.59 \\
\hline 4 & 98.29 & 55.49 & 92.31 & 54.29 & 52.71 & 56.93 & 73.33 & 9.6 & 46.14 & 71.66 & 80.8 & 62.94 & .39 & 59.83 & 53.03 \\
\hline $\mathrm{r}$ & 10.55 & 5.75 & 9.81 & 5.38 & 5.47 & 6 & 8 & 1.37 & 6.46 & 9.86 & 11.23 & 6.86 & .54 & 6.5 & 5.79 \\
\hline & .32 & 22.77 & 39.8 & 19.46 & 20.95 & 23.27 & 31.53 & & 21.96 & 34 & & .44 & & 26.25 & 23.17 \\
\hline & & & & & & & & & 4.92 & & 96 & 5.98 & - & .78 & 5.09 \\
\hline & 1.28 & 0.78 & 1.36 & 0.49 & 0.79 & 0.81 & 0.79 & 0.17 & 0.42 & 0.79 & 1.13 & 0.57 & 0.38 & 0.82 & 0.61 \\
\hline $\mathrm{Gd}$ & .43 & 3.44 & 6.24 & 2.6 & 3.18 & 3.81 & 4.19 & 1.04 & 3.31 & 4.5 & 6.21 & 4.38 & .83 & 4.65 & 3.67 \\
\hline$\Gamma \mathrm{b}$ & 93 & 0.5 & 94 & 0.32 & 0.4 & 5 & 0.48 & 9 & 0.71 & 82 & 1.46 & .59 & & 0.73 & 0.48 \\
\hline$y$ & & 3 & & & 2.36 & & & & 3.05 & & 7.01 & & & 4.2 & 2.2 \\
\hline [o & & 0.28 & 0.52 & & & 0.23 & 0.15 & 0.23 & 0.43 & 0.41 & 1.07 & 0.21 & 0. & 0. & 0.17 \\
\hline $\mathrm{Er}$ & 55 & 0.89 & 1.51 & 0.36 & 0.56 & 0.58 & 0.39 & 0.64 & 0.97 & 0.9 & 2.63 & 0.49 & 0.3 & 1.04 & 0.39 \\
\hline $\mathrm{Tm}$ & .17 & 0.12 & 0.17 & 0.03 & 0.06 & 0.06 & 0.04 & 0.11 & 0.13 & 0.11 & 0.34 & 0.04 & 0.03 & 0.11 & 0.03 \\
\hline $\mathrm{Yb}$ & & 1.21 & & & & & & & 0.81 & & 2.06 & & & 0.94 & 0.2 \\
\hline $\mathrm{Lu}$ & 0.24 & 0.2 & 0.21 & 0.05 & 0.08 & 0.09 & 0.06 & 0.11 & 0.11 & 0.09 & 0.27 & 0.04 & 0.04 & 0.13 & 0.04 \\
\hline$\Sigma$ REE & 225.94 & 125.73 & 214.03 & 116.81 & 118.49 & 129.15 & 162.1 & 25.22 & 106.8 & 161.91 & 192.51 & 143 & 54.3 & 140.3 & 120.56 \\
\hline $\mathrm{Eu}^{*}$ & 27.3 & 14.7 & 26.7 & 10.9 & 13.9 & 16.4 & 18.3 & 4.7 & 15.2 & 21.3 & 28.1 & 19.3 & 8 & 19.5 & 16.3 \\
\hline & 21 & 13.9 & 22.8 & 59.6 & 34.8 & 33 & 60.9 & 3.2 & 16.4 & 32.1 & 11.9 & 78.9 & 29.5 & 22.9 & 66. \\
\hline & 15.8 & 11.9 & 16.3 & 44 & 23.9 & 25.4 & 43.3 & 3.2 & 14.7 & 29.5 & 10.2 & 49.5 & 21.6 & 16.5 & 54.9 \\
\hline $\mathrm{Eu}_{\mathrm{N}} / \mathrm{Eu}^{*}$ & 0.54 & 0.61 & 0.59 & 0.52 & 0.65 & 0.57 & 0.5 & 0.42 & 0.32 & 0.43 & 0.46 & 0.34 & 0.55 & 0.48 & 0.4 \\
\hline
\end{tabular}

$\mathrm{Rb}, \mathrm{Sr}, \mathrm{Y}, \mathrm{Zr}, \mathrm{Nb}$ and $\mathrm{Ba}$ are XRF analysed, and other elements are ICP-MS analysed; La to Lu are ICP-MS analysed; Chondrite normalised values are taken after Taylor and McLennan $(1985) ; \mathrm{Eu}^{*}=\sqrt{ }\left(\mathrm{Sm}_{\mathrm{N}}\right) \times\left(\mathrm{Gd}_{\mathrm{N}}\right) ; \Sigma \mathrm{REE}=\mathrm{Sum}$ of total REE.

attributed to the source regions, and is consistent with the range of MS values obtained from the GGn, EPG and TLg that happen to be identical to ilmenite series granites (Ishihara 1977; Ishihara et al. 2002). The muscovites from GGn, EPG and TLg are celadonite to ferro-celadonite and are also typical to peraluminous felsic melts (e.g., Benincasa et al. 2003). Thus the micas (biotite and muscovite) in these felsic rocks represent primary liquidus phases and not the restite or secondary phases.

\subsubsection{Assessing the protoliths of the felsic magmas}

All three granite suites are peraluminous; the GGn (Av. Alumina Saturation Index, ASI = 1.13),
EPG (Av. ASI = 1.10) and TLg (Av. ASI = 1.31) are similar to the Early Palaeozoic granitoids of Lesser Himalayan belts of Nepal-India-Pakistan (Barbarin 1996), which evolved by fractional differentiation following partial melting, and resulted in a moderate peraluminousity (figure 14). The potential source rocks from which peraluminous felsic partial melts were generated can be very well constrained by comparison with the composition of experimental felsic melts formed by mica dehydration of metapelites, metagraywackes and muscovite-rich metapelites (Patiño Douce 1999; Lee et al. 2003). Although it is difficult to choose certain samples to represent the initial melt (or starting melt=anatectic melt), the average of GGn 
Table 8(b). Trace element (ppm) and elemental ratios of Early Palaeozoic granitoids (EPG).

\begin{tabular}{|c|c|c|c|c|c|c|c|c|c|c|c|c|c|c|c|}
\hline Sample no. & A1 & A2 & A3 & $\mathrm{A} 4$ & A 6 & $\mathrm{C} 1$ & $\mathrm{C} 7$ & C9 & $\mathrm{C} 10$ & C19 & $\mathrm{C} 23$ & $\mathrm{C} 27$ & $\mathrm{~S} 1$ & $\mathrm{R} 2$ & $\mathrm{R} 6$ \\
\hline & 6.1 & 16.5 & 7.5 & 20.3 & 17.6 & 15.5 & 15.7 & 13.1 & 15.3 & 17.5 & 20.5 & & 18.0 & 19.8 & 18.1 \\
\hline & 25.4 & 231.3 & 232.8 & 173.2 & 136.5 & 217.8 & & 311.8 & 293.0 & 330.4 & & & & & 189.2 \\
\hline & 94.5 & 103.5 & 96.9 & 122.1 & 125.7 & 119.9 & 61.5 & 54.7 & 92.0 & 63.2 & 54.0 & 93.1 & 95.4 & 127.9 & 149.6 \\
\hline & 25.7 & 23.2 & 38.4 & 15.7 & 24.7 & & 27.4 & 31.0 & 44.0 & 23.6 & & 42.0 & 34.3 & 17.2 & 33.0 \\
\hline & 153.4 & 158.5 & 168.8 & 250.1 & 196.5 & 161.4 & 102.1 & 102.3 & 293.0 & 144.4 & 111.0 & 298.1 & 258.6 & 158.0 & 206.0 \\
\hline & 94 & 9.6 & & 13.2 & 10.3 & & 7.8 & 6.5 & 17.0 & 12.2 & 9.0 & 16.6 & 13.9 & & 11.5 \\
\hline & 48.4 & 710.7 & 856.2 & 233.6 & 673.3 & 766.6 & 251.1 & 207.4 & 487.0 & 369.7 & 209.0 & 443.9 & 601.0 & 344.3 & 836.0 \\
\hline & & & & 1.2 & 1.1 & 0.8 & & 1.2 & 1.3 & 1.9 & 1.6 & 2.3 & 1.3 & 1.5 & 1.3 \\
\hline & 5.5 & 13.2 & 21.0 & 17.9 & 20.7 & 24.6 & 12.3 & 5.8 & 11.2 & 17.1 & 26.9 & 10.8 & 22.0 & 17.5 & 22.3 \\
\hline & & & & & 2.0 & & & 1.8 & 4.7 & 4.1 & 4.3 & 2.3 & 1.7 & & 2.6 \\
\hline & 72.0 & 182.0 & 173.0 & 122.0 & 210.0 & 228.0 & 139.0 & 140.0 & 119.0 & 128.0 & 140.0 & 118.0 & & 197.0 & 195.0 \\
\hline$r$ & .4 & & 2 & 1.4 & 1.4 & & & 5.7 & 3.2 & 5.2 & 5.8 & 3.1 & 2.5 & 1.5 & 1.3 \\
\hline & & & & & 0.2 & & & 1.5 & 0.6 & 0.9 & 1.5 & 0.7 & 0.4 & 0.6 & 0.2 \\
\hline & & & & & & & & 0.3 & 0.2 & 0.2 & 0.3 & 0.2 & .2 & 0.4 & 0.2 \\
\hline $\mathrm{i} / \mathrm{P}$ & & & & & & & & 2.3 & 6.3 & 3.2 & 2.6 & 5.4 & 6 & & 6.5 \\
\hline $\mathrm{a}$ & 30.59 & 21.69 & 37.08 & 36.95 & 36.17 & 33.27 & 14.81 & 13.17 & 13.38 & 22.67 & 41.91 & 32.12 & 32.83 & & 36.37 \\
\hline & 64 & 55.77 & 53 & 93.84 & 91.31 & 87 & 39.41 & 28.38 & 35.9 & 60.46 & 10 & 67.7 & 85 & & 93.97 \\
\hline & & & & 12 & 12.49 & & & 3.12 & 5.06 & .52 & & 7.45 & & & 13.13 \\
\hline & 7.42 & & & & 43.35 & & & 12.28 & 17.18 & & & 30.41 & & & 45.31 \\
\hline & & & & .05 & 8.97 & 9.0 & & 3.27 & 4.53 & 6.76 & & 6.61 & 8. & 5. & 10.05 \\
\hline & 81 & 05 & 28 & 1 & 1.39 & 1.3 & 0.6 & 0.38 & 0.51 & 0.74 & & 0.71 & 1. & 0.6 & 1.5 \\
\hline & & & & & .66 & & & 75 & 3.29 & & & 5.3 & & & 6.52 \\
\hline & & & & & & & & & 0.93 & & & 0.84 & & & 1.48 \\
\hline & .01 & 4.63 & 7.22 & 4.24 & 4.72 & 5.13 & 5.27 & 3.84 & 5.08 & 5.03 & 7.41 & 4.75 & 5.6 & 2.83 & 7.0 \\
\hline Io & 0.34 & .71 & 1.19 & 0.64 & 0.64 & 0.78 & 0.82 & 0.36 & 0.82 & 0.76 & 1.14 & 0.45 & 0.89 & 0.44 & 1.16 \\
\hline r & & & & & 1.47 & 2 & & 1.01 & 2.07 & 1.91 & & 1.31 & & 1.3 & 3.34 \\
\hline & & & & & & & & & & & & & & & 0.5 \\
\hline $\mathrm{Yh}$ & & & 2.93 & 1.22 & 0.95 & & & 1.03 & 1.84 & 1.94 & & 1.32 & 2.1 & 1.38 & 3.2 \\
\hline $\mathrm{Lu}$ & 0.09 & 0.23 & 0.43 & 0.18 & 0.11 & 0.26 & 0.25 & 0.15 & 0.25 & 0.27 & 0.37 & 0.2 & 0.3 & 0.19 & 0.4 \\
\hline & 147.42 & 133.05 & 220.02 & 208.64 & 208.81 & 201.59 & 98.66 & 70.24 & 91.14 & 144.01 & 257.35 & 159.4 & 197.65 & 139.97 & 224.11 \\
\hline $\mathrm{Eu}$ & 20.1 & 18.8 & 28.1 & 24.5 & 26.8 & & 15.5 & 11.3 & 14.5 & 20.8 & 35 & 22.4 & 25.9 & 14.9 & 30.5 \\
\hline & 35.3 & & 9 & 21.3 & 34.1 & 13.3 & & 9.1 & 5.6 & 8.7 & 11.8 & 16.7 & 11.4 & 14.3 & 8.2 \\
\hline$(\mathrm{Ce} / \mathrm{Yb})_{\mathrm{N}}$ & 24.1 & 8.6 & 8.3 & 19.9 & 24.9 & 12.9 & 5.4 & 7.1 & 5.1 & 8.1 & 10.7 & 13.3 & 10.6 & 11.9 & 7.5 \\
\hline $\mathrm{Eu}_{\mathrm{N}} / \mathrm{Eu}^{*}$ & 0.46 & 0.64 & 0.52 & 0.52 & 0.6 & 0.59 & 0.48 & 0.39 & 0.4 & 0.41 & 0.35 & 0.36 & 0.49 & 0.52 & 0.57 \\
\hline
\end{tabular}

$\mathrm{Rb}, \mathrm{Sr}, \mathrm{Y}, \mathrm{Zr}, \mathrm{Nb}$ and $\mathrm{Ba}$ are XRF analysed, and other elements are ICP-MS analysed; La to Lu are ICP-MS analysed; Chondrite normalised values are taken after Taylor and McLennan (1985); $\mathrm{Eu}^{*}=\sqrt{ }\left(\mathrm{Sm}_{\mathrm{N}}\right) \times\left(\mathrm{Gd}_{\mathrm{N}}\right) ; \Sigma \mathrm{REE}=\mathrm{Sum}$ of total REE.

samples, and EPG (R6) and TLg (TLg 2a) samples free from cumulate textures without the least silica contents in their groups, have been assumed to represent parental melt compositions and compared with experimental melt compositions. The GGn (av.) and EPG (R6) melts have compositions similar to those commonly produced from biotite-rich metapelite and metagraywacke sources (figure 16). Thus, in the generation of GGn and EPG melts, the contributions of Proterozoic metasedimentary sources can be very well recognised as also suggested for Early Palaeozoic granitoids of other Himalayan segments (e.g., Kumar et al. 1996; Valdiya 1995; Islam et al. 2005).

Himalayan TLg (TLg 2a) lie in the field of melt compositions formed from metagraywacke source, and a few TLg evolved to reach the compositional field of melts formed by partial melting of muscovite-rich metapelite (figure 16). It has been suggested that some TLg from other regions of the Himalaya have formed by muscovite dehydration melting of metapelites at $\mathrm{P} \sim$ $300 \mathrm{MPa}$ and $\mathrm{T} \geq 600-710^{\circ} \mathrm{C}$ (Visonā and Lombardo 2002). Searle (1999) opined that melting of pelitic protoliths could have occurred through muscovite breakdown, either during fluid-absent partial melting at $<750^{\circ} \mathrm{C}$, or by fluid-present partial melting at $<650^{\circ} \mathrm{C}$. Kawakami and Kobayashi (2006) inferred that the production of tourmaline leucogranite is limited to low degrees of partial melting $(\sim 10 \mathrm{wt} \%)$, regardless of the $\mathrm{P}-\mathrm{T}$ conditions and the reaction by which it was produced. 

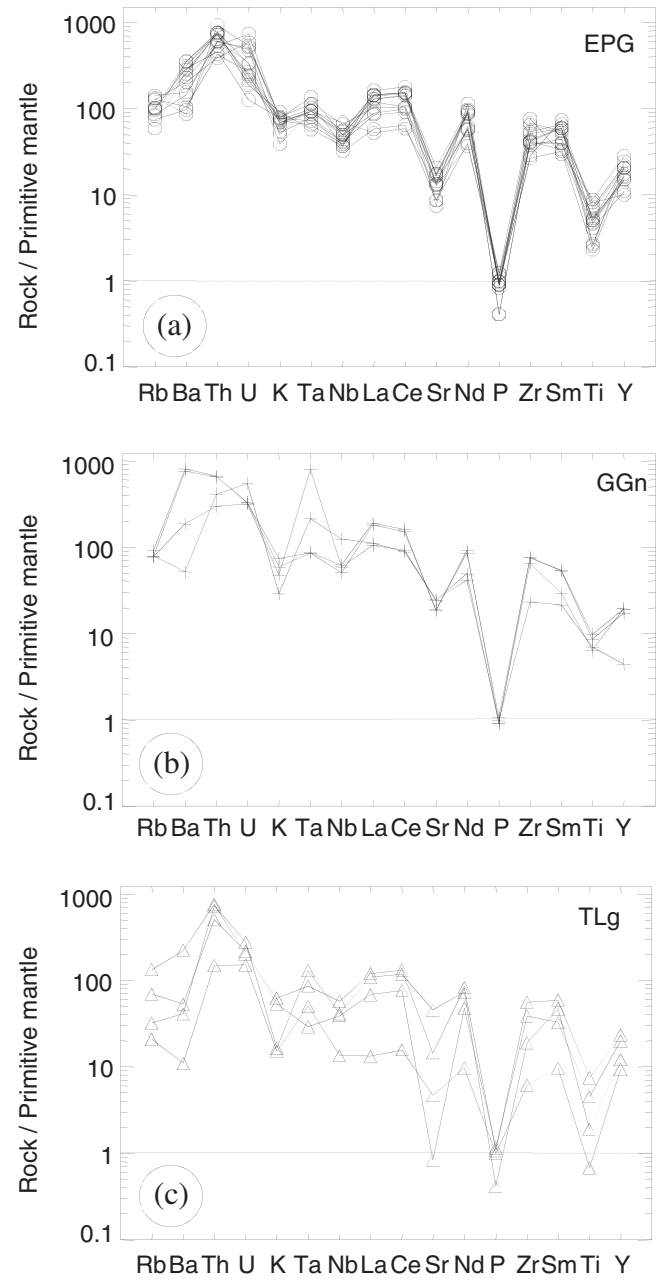

Figure 12. Primitive mantle (Sun and Mc Donough 1989) normalised trace element variation diagrams for (a) EPG, (b) GGn and (c) TLg.

However, partial contribution from the proximal protoliths like GGn and EPG during Himalayan collision tectonics to produce TLg melt cannot be entirely ruled out.

Comparing the alumina saturation index (ASI) of EPG ( $\ddot{\mathrm{x}}=1.10, \mathrm{n}=16)$ and $\mathrm{GGn}(\ddot{\mathrm{x}}=1.13, \mathrm{n}=$ $6)$ with that of mean ASI values of anatectic granites used in the melting experiments (compilation of Acosta-Vigil et al. 2003; table 9), it is suggested that the EPG and GGn melts were generated by fluid absent biotite melting. The ASI of EPG and GGn are however lower than the experimentally predicted ASI values $(\geq 1.20)$, which indicates that the EPG and GGn melts were undersaturated in aluminous minerals or failed to achieve equilibrium (Acosta-Vigil et al. 2003). The ASI of TLg ( $\ddot{\mathrm{x}}=$ $1.31, \mathrm{n}=9$ ) compares well with upper limit of experimentally predicted ASI values (1.15-1.30) but higher than the mean ASI value $(\ddot{\mathrm{x}}=1.17$, $\mathrm{n}=45)$ noted for other Himalayan leucogranites (Le Fort 1981; Searle and Fryer 1986; Stern et al. 1989; Scaillet et al. 1990; Guillot and Le Fort 1995;
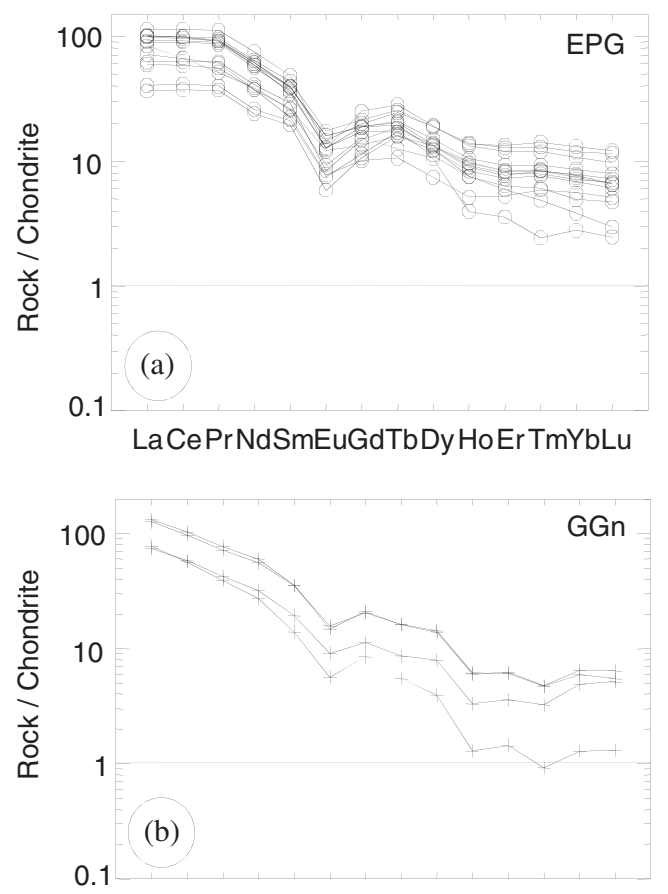

LaCePrNdSmEuGdTbDyHo ErTmYbLu

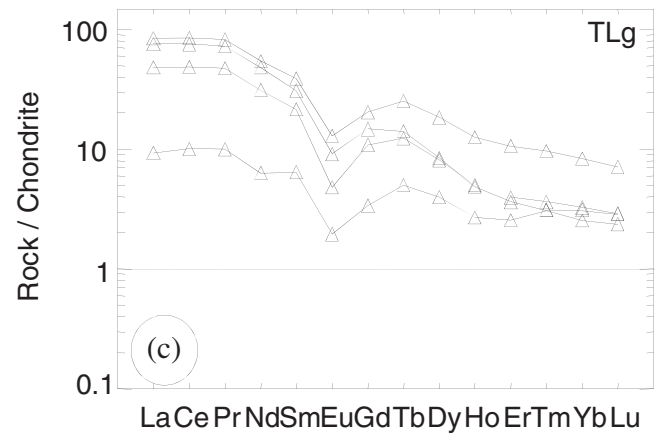

Figure 13. Chondrite normalised (normalisation values from Taylor and McLennan 1985) REE patterns for (a) EPG, (b) GGn and (c) TLg.

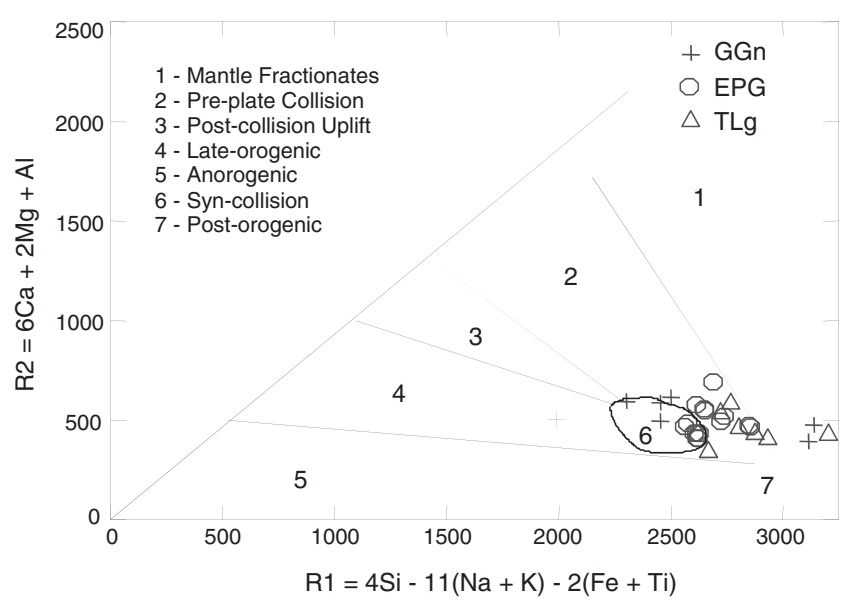

Figure 14. $\mathrm{R}_{1}-\mathrm{R}_{2}$ multicationic diagram (De la Roche et al. 1980) showing compositional fields for felsic rocks from different tectonic settings (Batchelor and Bowden 1985) and samples from GGn, EPG and TLg. The encircled field 6 represents the field of anatectic granites. 


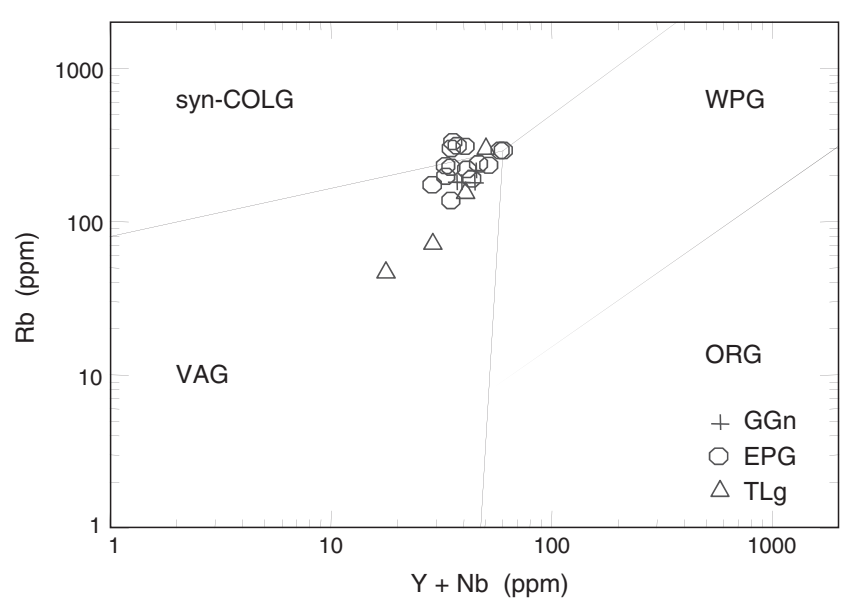

Figure 15. Tectonic discrimination for granites from Pearce et al. (1984) with samples from GGn, EPG and TLg.

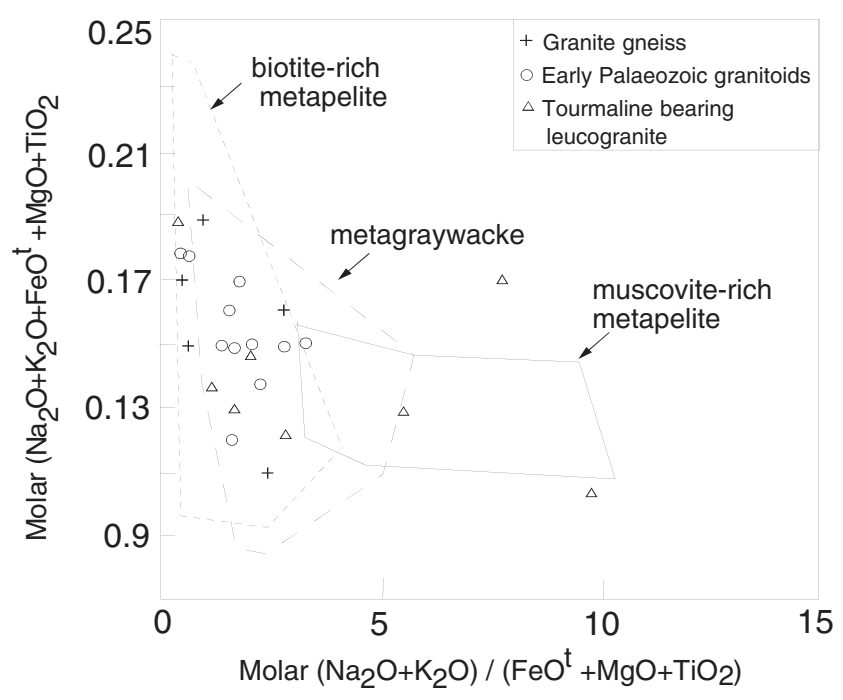

Figure 16. Chemical compositions of GGn, EPG and TLg compared with partial melts obtained from experimental partial melting studies of mica dehydration melting in metapelitic and metagraywacke protoliths. Fields of experimental melt compositions are shown from compilation of Patiño Douce (1999) and Lee et al. (2003).
Searle et al. 1997), which suggests likely generation of TLg melt by fluid-absent muscovite melting.

\subsubsection{Elemental variations and process diagnosis}

- Granite gneiss (GGn)

Average composition of GGn has been considered equivalent in parental magma composition, which subsequently evolved through fractional differentiation mechanism forming the GGn rock suite. $\mathrm{CaO}, \mathrm{K}_{2} \mathrm{O}, \mathrm{TiO}_{2}$ and $\mathrm{Fe}_{2} \mathrm{O}_{3}^{\mathrm{t}}$ behave as compatible elements during the crystallisation of felsic magmas most probably as a result of fractional crystallisation of biotite ( \pm muscovite), feldspars, $\mathrm{Fe}-\mathrm{Ti}$ oxides and apatite. Negative anomalies for $\mathrm{P}$ and Ti suggest that apatite and ilmenite played an important role during the evolution of GGn. Variation in Ba and $\mathrm{K}$ contents suggest a prime role for mica during crystallisation. Lesser variation in $\mathrm{Nb}$ suggests retention of Nb-hosting phases in the residue during crystal melting event.

- Early Palaeozoic granitoids (EPG)

The linear variation of $\mathrm{TiO}_{2}, \mathrm{Al}_{2} \mathrm{O}_{3}, \mathrm{Fe}_{2} \mathrm{O}_{3}$ and $\mathrm{CaO}$ against $\mathrm{SiO}_{2}$ suggests a low degree of fractional differentiation. The variation in $\mathrm{Sr}$ contents supports the early crystallisation of Ca-rich phases, but Sr cannot be fully accommodated in calcic-poor plagioclase $\left(\mathrm{An}_{15-33}\right)$ because of preferential partitioning of $\mathrm{Sr}$ and $\mathrm{Eu}$ in anorthiterich plagioclase (bytownite) which occurs at low oxygen fugacity conditions (e.g., Drake 1975, Blundy and Shimizu 1991). Rb variation suggests preferential partitioning and substitution in $\mathrm{K}$ bearing fractionating phases such as K-feldspar, biotite and muscovite. A negative Sr-anomaly (figure 11i) suggests the fractionation of plagioclase which is further corroborated by negative $\mathrm{Eu}$ anomaly. It is more likely that EPG parental melt might have been derived from plagioclase depleted source. Anomalously high content of Y

Table 9. Single-stage major elements based least-squares fractionation solution models of granite gneiss (GGn), Early Palaeozoic granitoids (EPG) and tourmaline bearing leucogranite (TLg).

\begin{tabular}{|c|c|c|c|c|c|c|}
\hline \multirow[b]{2}{*}{ Parent felsic magma } & \multicolumn{3}{|c|}{ Fractionating minerals (\%) } & \multirow[b]{2}{*}{$R^{2}$} & \multirow[b]{2}{*}{$\mathrm{F}$} & \multirow[b]{2}{*}{ Residual felsic magma } \\
\hline & $\begin{array}{l}\text { K-feldspar } \\
\quad(\mathrm{Kf})\end{array}$ & $\begin{array}{l}\text { Plagioclase } \\
(\mathrm{Pl})\end{array}$ & $\begin{array}{l}\text { Biotite } \\
\text { (Bt) }\end{array}$ & & & \\
\hline $\begin{array}{l}\text { Granite gneiss } \\
\left(\text { Average } \mathrm{GGn}, \mathrm{SiO}_{2}=70.80 \mathrm{wt} \%\right)\end{array}$ & 3.8 & 6.9 & 1.9 & 0.84 & 87.4 & $\mathrm{G} 4\left(\mathrm{SiO}_{2}=73.00 \mathrm{wt} \%\right)$ \\
\hline $\begin{array}{l}\text { Early Palaeozoic granitoids } \\
\left(\mathrm{R} 6, \mathrm{SiO}_{2}=70.83 \mathrm{wt} \%\right)\end{array}$ & 5.5 & 5.2 & 5.8 & 1.26 & 83.5 & $\mathrm{~A} 6\left(\mathrm{SiO}_{2}=74.35 \mathrm{wt} \%\right)$ \\
\hline $\begin{array}{l}\text { Tourmaline bearing leucogranite } \\
\left(\mathrm{TLg} 2 \mathrm{a}, \mathrm{SiO}_{2}=74.64 \mathrm{wt} \%\right)\end{array}$ & 6.2 & 2.5 & 3.5 & 0.15 & 87.2 & TLg 2c $\left(\mathrm{SiO}_{2}=77.67 \mathrm{wt} \%\right)$ \\
\hline
\end{tabular}

$R^{2}=$ sum of residual squares and $\mathrm{F}=$ percentage of residual felsic magma. 
in a few EPG may be due to partial dissolution of monazite and apatite present in the source prior to the crystallisation of EPG melt. The $\mathrm{Nb}, \mathrm{Zr}$ and $Y$ rich nature of EPG is most likely a result of HFSE-rich (residual) phases derived from melting of crustal source rocks. However, some primary zircons might have also crystallised in EPG magma.

- Tourmaline bearing leucogranite (TLg)

The variation of major elements in TLg appears to have been generated by partial melting of heterogeneous crustal sources followed by a low degree of fractional crystallisation. Partial melting of a crustal source would result in a minimum melt composition enriched in $\mathrm{Al}_{2} \mathrm{O}_{3}, \mathrm{~K}_{2} \mathrm{O}$ and $\mathrm{Na}_{2} \mathrm{O}$, whereas $\mathrm{Fe}_{2} \mathrm{O}_{3}^{\mathrm{t}}$, and $\mathrm{TiO}_{2}$ are at low concentrations, because of negligible contribution of these elements from metasedimentary (crustal) sources to the melt. The variation in $\mathrm{Zr}$, Ti and $\mathrm{Y}$ suggests a relatively minor role for zircon, whereas $\mathrm{Y}$ and $\mathrm{Ti}$ variations may account for early crystallisation of apatite and minor $\mathrm{Fe}-\mathrm{Ti}$ oxides.

\subsubsection{Modelling of major and trace including $R E E$ elements fractionation}

Petrological mixing calculations (Bryan et al. 1969) can be employed to model crystal fractionation of felsic plutons (e.g., Kumar and Kmet 1995; Lee et al. 2003). The basic principle is that fractionation of early-formed minerals from a parent magma will give rise to residual (daughter) magma. The average GGn composition, EPG (R6) and TLg (TLg 2a) samples that were free from cumulate-like texture and without least silica content were chosen as the parental felsic model melts. The average compositions of the fractionating phases and residual melts as granitoids with high $\mathrm{SiO}_{2}$ contents were used in calculations of several models of fractional crystallisation. The acceptable fractionation models in the evolution of GGn, EPG and TLg melts are summarised (table 9 ). In the model for GGn, about $13 \%$ fractionation of minerals (kf:pl:bt $=3.8: 6.9: 1.9$ ) from a GGn parent magma (average GGn) will produce about $87 \%$ of residual liquid (G4). In the EPG model, about $17 \%$ fractionation of mineral assemblage (kf:pl:bt $=5.5: 5.2: 5.8)$ from the EPG parental magma (R6) will produce $83 \%$ of residual melt (A6). In TLg model about $13 \%$ fractionation of mineral assemblage (kf:pl:bt $=$ 6.2:2.5:3.5) from TLg parent magma (TLg 2a) may produce $87 \%$ of residual melt (TLg 2c). The sum of residual squares $\left(R^{2}\right)$ obtained from these models is reasonably low and acceptable (GGn: $R^{2}=0.84$, EPG: $R^{2}=1.26$, TLg: $\left.R^{2}=0.15\right)$. Addition of magnetite-ilmenite to the fractionating assemblage
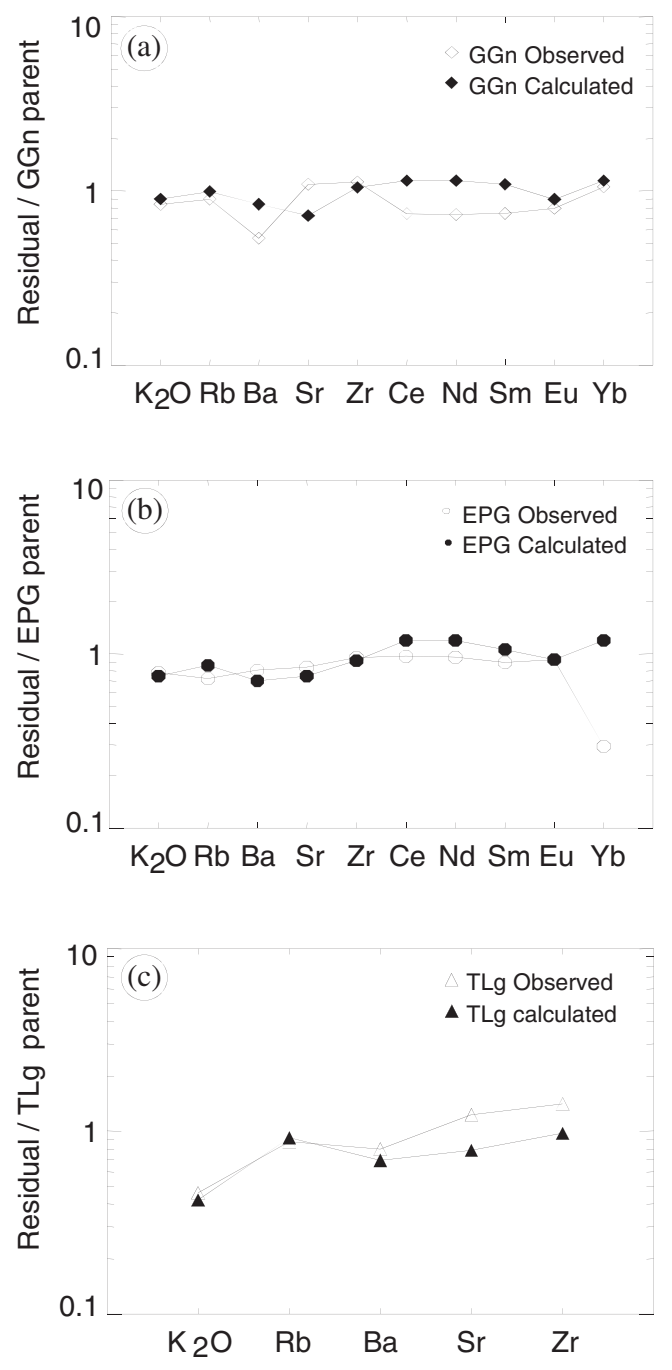

Figure 17. Normalised trace element spidergrams for felsic parents showing calculated and observed residual liquids derived from model Rayliegh fractionation of (a) GGn, (b) EPG and (c) TLg melts.

and a change of parental and residual compositions do not provide good statistical-fit results.

Trace element modelling of single-stage fractional crystallisation has been carried out for selected elements that are of petrogenetic significance using obtained proportions of fractionating minerals from their respective felsic parents, distribution coefficients $(K d)$ appropriate to felsic melts (Nash and Crecraft 1985) and the equation for Rayleigh fractionation (Gast 1968; Cox et al. 1979). Calculated and observed trace element patterns of residual liquids derived from the fractionation of respective GGn, EPG and TLg felsic parents are more-or-less similar (figure $17 \mathrm{a}-\mathrm{c}$ ). However, differences for certain elements such as Ce, $\mathrm{Nd}$, $\mathrm{Sm}$ for GGn, $\mathrm{Yb}$ for $\mathrm{EPG}$ and $\mathrm{Sr}$ and $\mathrm{Zr}$ for TLg, may be accounted for by either the use of an unrealistic $K d$ for these elements, or could be the result of accumulation and/or heterogeneous distribution effect of the accessory phases 
that host these elements during the fractional differentiation process. Since the feldspars $(\mathrm{pl}+\mathrm{kf})$ are the dominant phases to fractionate the REE patterns of EPG samples therefore exhibit negative $\mathrm{Eu}$ anomalies.

Low to moderately fractionated REE patterns with negative Eu anomalies for GGn and EPG suggest a significant role for the accessory phases and rock-forming minerals during melting and crystallisation events. Combined role of residual and fractionated plagioclase has also been considered a prime factor in the evolution of Proterozoic granitoids from the Lesser Himalaya (e.g., Miller et al. 2000). The sum of REE in TLg is much lower (up to $193 \mathrm{ppm}$ ) compared to that of leucogranite suite II of Bandal felsic magmatism (upto 270 ppm; Sharma and Rashid 2001), but is identical to other Himalayan leucogranites (Vidal et al. 1982; Scaillet et al. 1990). Thorpe et al. (1990) suggested that the low abundance of REE accompanied by a negative $\mathrm{Eu}$ anomaly may be the result of the fractionation of minor REE-bearing phases (e.g., apatite, xenotime, monazite and zircon) combined with that of plagioclase and K-feldspar. Depletion of LREE (La, Ce) in EPG, compared to GGn, may indicate either role of aqueous fluids or a feature inherited from source region.

\subsubsection{Relation to Pan-African felsic magmatism}

The GGn (601 $\pm 19 \mathrm{Ma})$ and EPG $(474 \pm 26 \mathrm{Ma})$ felsic magmatism relate to Neoproterozoic and Early Palaeozoic crustal events, respectively (e.g., Kröner 1984), which Kennedy (1964) described as the period of Pan-African crustal evolution, part of which is known as the East African Orogeny (EAO, 850-500 \pm 50 Ma; Stern 1994). The PanAfrican event marks the time span between the fragmentation of Rodinia and the formation of Greater Gondwana (Roy 1999), prior to Caledonide event (400 Ma, e.g., Chappell and Stephens 1988). The recognition of Pan-African events in the Indian shield, Sri Lanka and Africa, parts of which presently constitute the Kinnaur regions of Himalayan domain that are represented by felsic magmatisms (GGn and EPG), essentially mark the sites where the ocean closed and continental crust collided. Amalgamation of Gondwana (Pannotia) is one of the major pre-Pangean supercontinent events that occurred at ca $600 \mathrm{Ma}$, and break-up of Rodinia may have led to the assembly of Gondwana (Nance et al. 2013 and references therein).

\section{Conclusions}

Felsic magmatism in Kinnaur region of Himachal Higher Himalaya is represented by Neoproterozoic
$(601 \pm 19 \mathrm{Ma})$ granite gneiss (GGn), Early Palaeozoic (474 $\pm 26 \mathrm{Ma})$ granitoids (EPG), and tourmalinebearing Himalayan (14-28 Ma) leucogranites (TLg). The bulk magnetic susceptibility range for GGn $\left(\chi=0.168-0.190 \times 10^{-3} \mathrm{SI}\right), \mathrm{EPG}(\chi=$ $\left.0.096-0.130 \times 10^{-3} \mathrm{SI}\right)$, and TLg $(\chi=0.054-0.156 \times$ $10^{-3} \mathrm{SI}$ ) indicates that the conditions for crustal anatexis became increasingly reduced from preHimalayan to Himalayan felsic magmatic episodes. Modal compositions of GGn, EPG and TLg correspond to the granite (sensu stricto) melts generated by partial melting of fusible crustal components.

Plagioclase (GGn: $\mathrm{An}_{10}-\mathrm{An}_{31}$, EPG: $\mathrm{An}_{15}-\mathrm{An}_{33}$, TLg: $\mathrm{An}_{2}-\mathrm{An}_{15}$ ) and K-feldspar (GGn: $\mathrm{Or}_{88}-\mathrm{Or}_{95}$, EPG: $\left.\mathrm{Or}_{86}-\mathrm{Or}_{97}, \mathrm{TLg}: \mathrm{Or}_{87}-\mathrm{Or}_{94}\right)$ compositions suggest primary crystallisation from their respective peraluminous (S-type) felsic melts. Biotites from GGn $(100 * \mathrm{Fe} / \mathrm{Fe}+\mathrm{Mg}=55-66), \mathrm{EPG}(100 * \mathrm{Fe} / \mathrm{Fe}+$ $\mathrm{Mg}=53-73)$ and $\mathrm{TLg}(100 * \mathrm{Fe} / \mathrm{Fe}+\mathrm{Mg}=70-75)$ are ferribiotites enriched in siderophyllite exhibiting $3 \mathrm{Fe} \rightleftharpoons 2 \mathrm{Al}$ and $3 \mathrm{Mg} \rightleftharpoons 2 \mathrm{Al}$ substitutions typical of peraluminous (S-type), reduced felsic melts. Muscovites (ferro-celadonite) from GGn, EPG and TLg and tourmalines (schorl-elbaite) from EPG and TLg also suggest crystallisation from peraluminous, biotite-tourmaline and Li-poor felsic melts.

GGn and EPG parental melts appear to have derived from biotite-rich metapelite and metagraywacke sources; whereas TLg melt appear formed from relatively heterogeneous crustal sources during Himalayan orogenesis. Major elements modelling suggests low degree of fractional differentiation of felsic melts, i.e., ca 13\% fractionation of major rock-forming minerals (kf-pl-bt) from GGn parent produces $87 \%$ residual felsic liquid; ca $17 \%$ from EPG parent produces $83 \%$ of residual felsic melt and; ca $13 \%$ TLg parent produces $87 \%$ of residual felsic melt. Calculated and observed trace elements of residual felsic magmas derived from GGn, EPG and TLg parents are more-or-less similar, but discrepancies for some elements were most likely the result of heterogeneous mineral distribution or sorting during fractional differentiation.

\section{Acknowledgements}

The research work was partly supported by DST-New Delhi grant and partly by INSA-JSPS Fellowship awarded to Santosh Kumar (ID No. AP210219004). Prof Hisao Tanaka is thanked for continuing support in this study at Yamagata University, Japan. Dr V Balaram is thanked for extending ICP-MS analytical facility at National Geophysical Research Institute, Hyderabad, India. 
Dr Tamal K Ghosh helped with electron microprobe analysis at Indian Institute of Technology, Roorkee. E W Sawyer is highly thanked for constructive scientific comments and correction of language. Comments from two anonymous reviewers have greatly improved the earlier version. Rajesh $\mathrm{K}$ Srivastava is thanked for scientific and editorial remarks.

\section{References}

Abdel-Rahman A M 1994 Nature of biotites from alkaline, calc-alkaline and peraluminous magmas; J. Petrol. 35 $525-541$.

Acosta-Vigil A, London D, Morgan VI G B and Dewers T A 2003 Solubility of excess alumina in hydrous granitic melts in equilibrium with peraluminous minerals at 700 $800^{\circ} \mathrm{C}$ and $200 \mathrm{MPa}$, and applications of the alumina saturation index; Contrib. Mineral. Petrol. 146 100-119.

Balaram V and Gnaneshwar Rao T 2003 Rapid determination of REEs and other trace elements in geological samples by microwave acid digestion and ICP-MS; Atomic Spectros. 24 206-212.

Balaram V, Ramesh S L and Anjaiah K V 1996 New trace element and REE data in thirteen GSF reference samples by ICP-MS; Geostand. Newslett. 20 71-78.

Ban M, Takahashi K, Horie T and Toya N 2005 Petrogenesis of mafic inclusions in rhyolitic lavas from the Narugo volcano, Japan; J. Petrol. 46 1544-1563.

Barbarin B 1996 Genesis of two main types of peraluminous granitoids; Geology 24 295-298.

Bartarya S K, Virdi N S and Sah M P 1996 Landslides hazards: Some case studies from the Satluj valley, Himachal Pradesh; Him. Geol. 17 193-207.

Bassi U K and Chopra S 1983 A contribution to the geology of Kinnaur Himalaya, Himachal Pradesh; Indian J. Earth Sci. 10 96-99.

Batchelor R A and Bowden P 1985 Petrogenetic interpretation of granitoid rock series using multicationic parameters; Chem. Geol. 48 43-55.

Beane R E 1974 Biotite stability in the porphyry copper environment; Econ. Geol. 69 241-256.

Benincasa E, Brigatti M F, Poppi L and Barredo F B 2003 Crystal chemistry of dioctahedral micas from peraluminous granites: The Pedrobernardo pluton (central Spain); Eur. J. Mineral. 15 543-550.

Bhargava O N 1982 The tectonic windows of the Lesser Himalaya; Him. Geol. 10 135-155.

Bhargava O N, Bassi U K and Sharma R K 1991 The crystalline thrust sheets, age of metamorphism, evolution and mineralization of the Himachal Himalaya; Indian Mineral. $451-18$.

Blundy J D and Shimizu N 1991 Trace element evidence for plagioclase recycling in calc alkaline magmas; Earth Planet. Sci. Lett. (Amsterdam) 102 178-197.

Bryan W B, Finger L W and Chayes F 1969 Estimating proportions in petrographic mixing equations by leastsquares approximation; Science 163 926-927.

Burkhard D J M 1991 Temperature and redox path of biotite-bearing intrusives: A method of estimation applied to S- and I-type granites from Australia; Earth Planet. Sci. Lett. 104 89-98.

Chappell B W and Stephens W E 1988 Origin of infracrustal (I-type) granite magmas; Trans. Royal Soc. Edin., Earth Sci. 79 71-86.

Chappell B W and White A J R 1974 Two contrasting granite types; Pacific Geol. 8 173-174.
Clarke B D 1992 Granitoid rocks; Chapman and Hall, London, 283p.

Collins W J and Sawyer E W 1996 Pervasive granitoid magma transfer through the lower-middle crust during non-coaxial compressional deformation; J. Metamorph. Geol. 14 565-579.

Cox K G, Bell J D and Pankhurst R J 1979 The interpretation of igneous rocks; George Alien and Unwin, London, $450 \mathrm{p}$.

De Albuquerque C A R 1973 Geochemistry of biotites from granitic rocks, northern Portugal; Geochim. Cosmochim. Acta 37 1777-1802.

De la Roche H, Leterrier J, Grandclaude P and Marchal M 1980 A classification of volcanic and plutonic rocks using $\mathrm{R}_{1}-\mathrm{R}_{2}$ diagrams and major element analysis - its relationship with current nomenclature; Chem. Geol. 29 $183-210$.

Debon F and Le Fort P 1983 A chemical-mineralogical classification of common plutonic rocks and associations; Trans. Royal Soc. Edin., Earth Sci. 73 135-149.

Deer W A, Howie R A and Zussman J 1963 Rock-forming minerals, Sheet silicates; Longmans, Green and Co., London 3, 270p.

Drake M J 1975 The oxidation state of europium as a indicator of oxygen fugacity; Geochim. Cosmochim. Acta 39 $55-64$.

Dymek R F 1983 Titanium, aluminium and interlayer cation substitutions in biotite from high grade gneisses, west Greenland; Am. Mineral. 68 880-899.

Foit F F and Rosenberg P E 1979 The structure of vanadium-bearing tourmaline and its implications regarding tourmaline solid solutions; Am. Mineral. 64 788-798.

Foster M D 1960 Interpretation of the composition of trioctahedral micas; U.S. Geol. Surv. Prof. Paper 354B 1-49.

Gansser A 1964 Geology of the Himalaya; Interscience Publ., John Wiley and Sons Ltd., London, 289p.

Gast P W 1968 Trace element fractionation and origin of tholeiitic and alkaline magma types; Geochim. Cosmochim. Acta 32 1057-1176.

Guillot S and Le Fort P 1995 Geochemical constraints on the bimodal origin of High Himalayan leucogranites; Lithos 35 221-234.

Gupta V, Virdi N S and Prakash S 2001 Morphometric assessment of active landslides in the higher Himalayan crystallines, Satluj valley, Himachal Pradesh (India); Him. Geol. 22 99-107.

Harris N B W and Inger S 1992 Trace element modelling of pelite derived granitoids; Contrib. Mineral. Petrol. 110 $46-56$.

Hawthorne F C and Henry D J 1999 Classification of the minerals of the tourmaline group; Eur. J. Mineral. 11 201-215.

Henry D J and Guidotti C V 1985 Tourmaline as a petrogenetic indicator mineral: An example from the staurolitegrade metapelites of NW Maine; Am. Mineral. 70 1-15.

Ishihara S 1977 The magnetite-series and ilmenite-series granitic rocks; Mining Geol. 27 293-305.

Ishihara S 1984 Granitoid series and Mo/Sn-W mineralization in east Asia; Geol. Surv. Japan Rep. 263 173-208.

Ishihara S and Matsuhisa Y 1999 Oxygen isotopic constraints on the genesis of the Miocene Outer Zone in Japan; Lithos 46 523-534.

Ishihara S, Robb L J, Anhaeusser C R and Imai A 2002 Granitoid series in terms of magnetic susceptibility: A case study from the Barberton region, South Africa; Gondwana Res. 5 581-589.

Islam R and Gururajan N S 2003 Geochemistry, petrogenesis and tectonic setting of Akpa-Rakcham granites of Sutlej valley, Himachal Pradesh, India; Him. Geol. 24 63-76. 
Islam R, Ahmad T and Khanna P P 2005 An overview on the granitoids of the NW Himalaya; Him. Geol. 26 49-60.

Kakar R K 1988 Geology and tectonic setting of central crystalline rocks of southern parts of Higher Himachal Himalaya; J. Geol. Soc. India 31 243-250.

Kawakami T and Kobayashi T 2006 Trace element composition and degree of partial melting of pelitic migmatites from the Aoyama area, Ryoke metamorphic belt, SW Japan: Implications for the source region of tourmaline leucogranites; Gondwana Res. 9 176-188.

Kennedy W Q 1964 The structural differentiation of Africa in the Pan-African $( \pm 500)$ tectonic episode; 8th annual report, Research Inst. African Geology, Univ. of Leeds, Leeds, $48 \mathrm{p}$.

Kretz R 1983 Symbols for rock forming minerals; $\mathrm{Am}$. Mineral. 68 277-279.

Kröner A 1984 Ophiolites and the evolution of tectonic boundaries in the Late Proterozoic Arabian-Nubian shield of northeast Africa and Arabia; Precamb. Res. 27 277-300.

Kumar S and Kmet J 1995 The calculated magma differentiation trend of the Hodruša-Štiavnica intrusive complex, western Carpathians; Bull. Czech. Geol. Surv. 70 15-18.

Kumar S, Singh B N and Joshi M 1996 Petrogenesis and tectonomagmatic environment of Cambro-Ordovician granitoids of Himalaya: A reappraisal; In: Proc. Sym. NW Himalaya and Foredeep; Geol. Surv. India Spec. Publ. 21 205-214.

Kwatra S K, Singh S, Singh V P, Sharma R K, Bimal Rai and Naval Kishor 1999 Geochemical and geochronological characteristics of the Early Palaeozoic granitoids from Sutlej-Baspa valleys, Himachal Himalayas; In: Geodynamics of the NW Himalaya (eds) Jain A K and Manickavasagam R M, Gond. Res. Group, Memoir 6 $145-158$.

Laymere J and Bowden P 1982 Plutonic rock type series: Discrimination of various granitoid series and related rocks; J. Volcanol. Geoth. Res. 14 169-186.

Le Fort P 1975 Himalayas: The collided range. Present knowledge of the continental arc; Am. J. Sci. 275 1-44.

Le Fort P 1981 Manaslu leucogranite: A collision signature of the Himalaya. A model for its genesis and emplacement; J. Geophys. Res. 86 10,555-10,568.

Le Fort P, Debon F, Pecher A, Sonet J and Vidal P 1986a The 500 Ma magmatic event in Alpine Southern Asia: A thermal episode at Gondwana scale; Sciences de la Terre, Memoir 47 191-209.

Le Fort P, Debon F and Sonet J 1986b The 'Lesser Himalayan' cordierite granite belt: Typology and age of the pluton of Manserah (Pakistan); Proc. Int. Geodynamic Conf., Peshawar; Univ. Peshawar Geol. Bull. 13 51-61.

Lee S-Y, Barnes C G, Howard K A, Frost C D and Snoke A W 2003 Petrogenesis of Mesozoic peraluminous granites in the Lamoille Canyon area, Ruby Mountains, Nevada, USA; J. Petrol. 44 713-732.

LeMaitre R W 2002 Igneous rocks: A classification and glossary of terms. Recommendations of the International Union of Geological Sciences; Subcommission on the Systematics of Igneous rocks. 2nd edn; Cambridge University Press, Cambridge, 236p.

London D and Manning D A C 1995 Chemical variation and significance of tourmaline from southwest England; Econ. Geol. 90 495-519.

Loomis T P 1982 Numerical simulations of crystallisation processes of plagioclase in complex melts: The origin of major and oscillatory zoning in plagioclase; Contrib. Mineral. Petrol. 81 219-229.
Mehnert K R 1968 Migmatites and the origin of granitic rocks; Dev. Petrol. 1, Elsevier, Amsterdam, 393p.

Mehta P K 1977 Rb-Sr geochronology of the Kulu-Mandi belt: Its implication for the Himalayan tectonogenesis; Geologischa Rundschau 66 156-288.

Miller C F, Stoddard E F, Bradfish L J and Dollase W A 1981 Composition of plutonic muscovite: Genetic implications; Can. Mineral. 19 25-34.

Miller C, Klötzli U, Frank W, Thöni M and Grasemann B 2000 Proterozoic crustal evolution in the NW Himalaya (India) as recorded by circa $1.80 \mathrm{Ga}$ mafic and $1.84 \mathrm{Ga}$ granitic magmatism; Precamb. Res. 103 191-206.

Mishra D K 1993 Tectonic setting and deformational features in Satluj and Beas valleys of Himachal Pradesh; Indian J. Petrol. Geol. 2 81-92.

Monier G and Robert J L 1986 Evolution of the miscibility gap between muscovite and biotite solid solutions with increasing lithium content: An experimental study in the system $\mathrm{K}_{2} \mathrm{O}-\mathrm{Li}_{2} \mathrm{O}-\mathrm{MgO}-\mathrm{FeO}-\mathrm{Al}_{2} \mathrm{O}_{3}-\mathrm{SiO}_{2}-\mathrm{H}_{2} \mathrm{O}-\mathrm{HF}$ at $600^{\circ} \mathrm{C}, 2$ kbar $\mathrm{P}_{\mathrm{H} 2 \mathrm{O}}$ : Comparison with natural lithium micas; Mineral. Mag. 50 641-651.

Murata M 1993 Major and trace elements analysis of Korea Institute of Energy and Resources igneous rock reference samples using X-ray fluorescence spectrometer; $J$. Naruto Coll. Edue 8 37-50 (in Japanese with English abstract).

Nance R D, Murphy J B and Santosh M 2013 The supercontinent cycle: A retrospective essay; Gondwana Res. $\mathbf{2 5}$ $4-29$.

Nash W P and Crecraft H R 1985 Partition coefficients for trace elements in silicic magmas; Geochim. Cosmochim. Acta 49 2300-2322.

Patiño Douce A E 1999 What do experiments tell us about the relative contributions of crust and mantle to the origin of granitic magmas? In: Understanding granites: Integrating new and classical techniques (eds) Castro A, Fernandes C and Vigneresse J, Geol. Soc. London Spec. Publ. 168 55-75.

Pearce J A, Harris N B W and Tindle A G 1984 Trace element discrimination diagrams for the tectonic interpretation of granitic rocks; J. Petrol. 25 956-983.

Roy A B 1999 Neoproterozoic crustal evolution and IndiaGondwana linkage - an epilogue; Gondwana Res. 2 193-198.

Scaillet B, France-Lanord C and Le Fort P 1990 BadrinathGangotri plutons (Garhwal, India): Petrological and geochemical evidence for fractionation processes in a High Himalayan leucogranite; J. Volcanol. Geotherm. Res. 44 $163-188$.

Searle M P 1999 Emplacement of Himalayan leucogranites by magma injection along giant sill complexes: Examples from the Cho Oyu, Gyachung Kang and Everest leucogranites (Nepal Himalaya); J. Asian Earth Sci. 17 773-783.

Searle M P and Fryer B J 1986 Garnet, tourmaline and muscovite bearing leucogranites, gneisses and migmatites of the Higher Himalayas from Zanskar, Kulu, Lahoul and Kashmir; In: Collision Tectonics (eds) Coward M P and Ries A C, Geol. Soc. Spec. Publ. 19 185-201.

Searle M P, Parrish R R, Hodges K V, Hurford A, Ayres M W and Whitehouse M J 1997 Shisha Pangma leucogranite, South Tibetan Himalaya: Field relations, geochemistry, age, origin and emplacement; J. Geol. 105 $295-317$.

Sharma K K 1976 A contribution to the geology of Satluj valley, Kinnaur, Himachal Pradesh, India; In: Colloques Inter. CNRS: Himalaya Science de la Terre, CNRS, Paris $268369-378$.

Sharma K K and Rashid S A 2001 Geochemical evolution of peraluminous Palaeoproterozoic Bandal orthogneiss, NW Himalaya, Himachal Pradesh, India: Implications for the 
ancient crustal growth in the Himalaya; J. Asian Earth Sci. 19 413-428.

Singh B and Kumar S 2005 Petrogenetic appraisal of Early Palaeozoic granitoids of Kinnaur district, Higher Himachal Himalaya, India; Gondwana Res. 8 67-76.

Slivko M M 1959 Manganese tourmalines; Mineral. Sborn. Lvov 13 139-148 (in Russian).

Srikantia S V and Bhargava O N 1998 Geology of Himachal Pradesh; Geol. Soc. India Memoir 9 Bangalore, 406p.

Stern R J 1994 Arc assembly and continental collision in the Neoproterozoic East African orogen: Implications for the consolidation of Gondwanaland; Ann. Rev. Earth Planet. Sci. 22 319-351.

Stern C R, Kligfield R, Schelling D, Virdi N S, Futa K, Peterman Z E and Amini H 1989 The Bhagirathi leucogranite of the High Himalaya (Garhwal, India): Age, petrogenesis and tectonic implications; Geol. Soc. Am. Spec. Paper 232 33-45.

Streckeisen A 1973 Plutonic rocks. Classification and nomenclature recommended by the IUGS Subcommission on the systematic of Igneous rocks; Geotimes 18(10) 26-30.

Stone M, Exley C S and George M C 1986 Composition of trioctahedral micas in the Cornubian batholith; Mineral. Mag. 52 175-192.

Sun S S and Mc Donough W F 1989 Chemical and isotopic systematics of oceanic basalts: Implications for mantle composition and processes; (eds) Saunders A D and Norry M S, In: Magmatism in ocean basins Geol. Soc. London Sec. Publ. 42 313-345.

Taylor S R and McLennan S M 1985 The continental crust: Its composition and evolution; Blackwell, Oxford, $295 \mathrm{p}$.

Thorpe R S, Tindle A G and Gledhill A 1990 The petrology and origin of the Tertiary Lundy granite (Bristol Channel, UK); J. Petrol. 31 1377-1406.

Tindle A G and Webb P C 1990 Estimation of lithium contents in trioctahedral micas using microprobe data: Application to micas from granitic rocks; Eur. J. Min. 2 595-610.

Tischendorf G 1997 Geochemical and petrographic characteristics of silicic magmatic rocks associated with rare metal mineralization (eds) Stempork M, Burnol L and Tischendorf G, In: Ustredni Ustav Geologicky Prague 2 41-98.
Tischendorf G, Förster H J and Gottesmann B 1999 The correlation between lithium and magnesium in trioctahedral micas: Improved equation for $\mathrm{Li}_{2} \mathrm{O}$ estimation from MgO data; Mineral. Mag. 63 57-74.

Torres-Ruiz J, Pesquera A and Sánches-Vizcaino L 2003 Chromian tourmaline and associated Cr-bearing minerals from the Nevado-Filábride Comples (Betic Cordilleras, SE Spain); Mineral. Mag. 67 517-533.

Valdiya K S 1995 Proterozoic sedimentation and PanAfrican geodynamic developments in the Himalaya; Precamb. Res. 74 35-55.

Vance J A 1965 Zoning in igneous plagioclase: Patchy zoning; J. Geol. 73 636-651.

Vernon R H 1986 K-feldspar megacrysts in granites-phenocrysts, not porphyroblasts; Earth Sci. Rev. 23 1-63.

Vidal P, Cocherie A and Le Fort P 1982 Geochemical investigation of the origin of the Manaslu leucogranite (Himalaya Nepal); Geochim. Cosmochim. Acta 46 2277-2292.

Visonā D and Lombardo B 2002 Two-mica and tourmaline leucogranites from the Everest-Makalu region (NepalTibet). Himalayan leucogranite genesis by isobaric heating; Lithos 62 125-150.

Wiebe R A 1968 Plagioclase stratigraphy: A record of magmatic conditions and events in a granitic stock; Am. J. Sci. 266 690-703.

Wones D R and Eugster H P 1965 Stability of biotite: Experiment, theory and application; Am. Mineral. 50 1222-1272.

Wyllie P J 1997 Crustal anatexis: An experimental review; Tectonophys. 43 41-71.

Yamada Y, Kohno H and Murata M 1985 A low dilution fusion method for major and trace element analysis of geological samples; Advances in X-ray Analysis 26 23-44 (in Japanese with English abstract).

Yavuz F 1997 Tourmal: software package for tourmaline, tourmaline-rich rocks and related ore deposits; Comput. Geosci. 23 947-959.

Yavuz F 2001 Limica: A program for estimating Li from electron microprobe mica analyses and classifying trioctahedral micas in terms of composition and octahedral site occupancy; Comput. Geosci. 27 215-227.

Yavuz F and Öztas T 1997 Bioterm: A program for evaluating and plotting microprobe analyses of biotite from barren and mineralized magmatic suites; Comput. Geosci. 23 897-907.

Yoder H J, Stewart D B and Smith J R 1957 Feldspars; Carnegie Inst. Wash. Year Book 56 206-214.

MS received 4 April 2016; revised 14 July 2016; accepted 18 July 2016

Corresponding editor: R K SRIVASTAVA 